\title{
Meiotic DNA double-strand breaks and chromosome asynapsis in mice are monitored by distinct HORMAD2- independent and -dependent mechanisms
}

\author{
Lukasz Wojtasz, ${ }^{1}$ Jeffrey M. Cloutier, ${ }^{2}$ Marek Baumann, ${ }^{1}$ Katrin Daniel, ${ }_{1}^{1}$ János Varga ${ }_{1}^{3}$ Jun Fu, ${ }^{4}$ \\ Konstantinos Anastassiadis, ${ }^{5}$ A. Francis Stewart, ${ }^{4}$ Attila Reményi, ${ }^{3}$ James M.A. Turner, ${ }^{2}$ \\ and Attila Tóth ${ }^{1,6}$ \\ ${ }^{1}$ Institute of Physiological Chemistry, Technische Universität Dresden, Dresden 01307, Germany; ${ }^{2}$ Divisional of Developmental
Genetics, MRC National Institute for Medical Research, London, NW7 1AA, United Kingdom; ${ }^{3}$ Department of Biochemistry,
Institute of Biology, Eötvös Loránd University, 1117 Budapest, Hungary; ${ }^{4}$ Genomics, ${ }^{5}$ Center for Regenerative Therapies
Dresden, BioInnovationsZentrum, Technische Universität Dresden, Dresden 01307, Germany
}

Meiotic crossover formation involves the repair of programmed DNA double-strand breaks (DSBs) and synaptonemal complex (SC) formation. Completion of these processes must precede the meiotic divisions in order to avoid chromosome abnormalities in gametes. Enduring key questions in meiosis have been how meiotic progression and crossover formation are coordinated, whether inappropriate asynapsis is monitored, and whether asynapsis elicits prophase arrest via mechanisms that are distinct from the surveillance of unrepaired DNA DSBs. We disrupted the meiosis-specific mouse HORMAD2 (Hop1, Rev7, and Mad2 domain 2) protein, which preferentially associates with unsynapsed chromosome axes. We show that HORMAD2 is required for the accumulation of the checkpoint kinase ATR along unsynapsed axes, but not at DNA DSBs or on DNA DSBassociated chromatin loops. Consistent with the hypothesis that ATR activity on chromatin plays important roles in the quality control of meiotic prophase, HORMAD2 is required for the elimination of the asynaptic Spo11 ${ }^{-/-}$, but not the asynaptic and DSB repair-defective $\mathrm{Dmc1}^{-/-}$oocytes. Our observations strongly suggest that HORMAD2-dependent recruitment of ATR to unsynapsed chromosome axes constitutes a mechanism for the surveillance of asynapsis. Thus, we provide convincing evidence for the existence of a distinct asynapsis surveillance mechanism that safeguards the ploidy of the mammalian germline.

[Keywords: asynapsis surveillance; meiotic prophase checkpoint; ATR; HORMA domain; meiosis; aneuploidy] Supplemental material is available for this article.

Received January 30, 2012; revised version accepted March 19, 2012.

Orderly chromosome segregation during the first meiotic division requires that homologous maternal and paternal chromosomes (called homologs) become physically linked, thereby forming so-called bivalents during the first meiotic prophase (Page and Hawley 2003). In most organisms, including mammals, meiotic recombination generates reciprocal exchanges, called crossovers (COs), between homologous DNA sequences. Interhomolog COs and sister chromatid cohesion together form the basis of the physical linkages, called chiasmata, that connect pairs of homologs during the first meiotic metaphase. Therefore, at least one $\mathrm{CO}$ must form between each pair of homologs to ensure correct segregation during the first meiotic division.

${ }^{6}$ Corresponding author.

E-mail attila.toth@mailbox.tu-dresden.de.

Article is online at http://www.genesdev.org/cgi/doi/10.1101/gad.187559.112.
$\mathrm{CO}$ formation requires that homologs find each other. To achieve this, double-strand breaks (DSBs) are actively introduced into the genome at the beginning of prophase by the SPO11 enzyme (Keeney et al. 1997; Baudat et al. 2000; Romanienko and Camerini-Otero 2000). This is followed by the resection of DSB ends, which creates $3^{\prime}$ ssDNA overhangs (Neale et al. 2005). The RAD51 and DMC1 recombinases bind single-stranded DSB ends and assist homology search through promoting strand invasion of resected DSB ends into homologous DNA sequences (Baudat and de Massy 2007). Several DSB ends work in parallel on each pair of homologs to ensure alignment along the full lengths of homologs.

Aligned sections of homologs become connected by the synaptonemal complex (SC), which consists of three parallel elongated elements: two axial elements (AEs), each formed by the shared axes of a sister chromatid pair, and 
a central element that is positioned between aligned AEs by transverse filaments that connect central elements to AEs (Page and Hawley 2003). The SC and/or its components are required for efficient $\mathrm{CO}$ formation and the efficient completion of DSB repair steps subsequent to homolog alignment (Page and Hawley 2003; Baudat and de Massy 2007). After SC formation, most DSBs become repaired from homologs as noncrossovers, and at least one DSB on each pair of homologs is turned into a CO. It is postulated that meiotic progression beyond prophase must be prevented until DSB repair, homolog alignment, and SC formation are completed to avoid formation of gametes with chromosome abnormalities. Indeed, in mammals, meiotic prophase quality-control mechanisms (hereafter called prophase checkpoints) eliminate meiocytes with defects in these processes during the first meiotic prophase (Barchi et al. 2005; Bolcun-Filas et al. 2007, 2009; Hamer et al. 2008a; Burgoyne et al. 2009), thereby minimizing the formation of gametes with an abnormal chromosome set or unrepaired DNA.

The DNA damage response (DDR) sensor kinase ATR has been implicated in the control of progression through meiotic prophase (Turner et al. 2004; Bellani et al. 2005; Mahadevaiah et al. 2008; Daniel et al. 2011). In particular, accumulation of ATR activity on unsynapsed chromatin regions, which are also often rich in unrepaired DSBs, is considered a crucial constituent of meiotic prophase checkpoint mechanisms in both sexes (Burgoyne et al. 2009). The DDR protein MDC1 promotes the accumulation of ATR activity on chromatin loops emanating from unsynapsed chromosome axes (Ichijima et al. 2011). It has been proposed that this process is preceded by MDC1-independent recruitment of ATR activity to unsynapsed chromosome axes and to unrepaired DSBs positioned along unsynapsed chromosome axes in meiotic prophase cells (Ichijima et al. 2011). ATR recruitment along unsynapsed sex chromosome axes in spermatocytes was shown to depend on the DDR protein BRCA1 (Turner et al. 2004). Nevertheless, the mechanism and the true significance of recruitment of ATR and other DDR components to unsynapsed chromosome axes have remained little understood. Likewise, it remained uncertain whether DSBs, unsynapsed chromosome axes, or both activate ATR-mediated checkpoint responses during meiosis.

We and others have identified two meiosis-specific HORMA (Hop1, Rev7, and Mad2) domain proteins, HORMAD1 and HORMAD2, that preferentially localize to unsynapsed chromosome axes in mice in both sexes (Wojtasz et al. 2009; Fukuda et al. 2010; Shin et al. 2010). HORMAD1 ensures that sufficient numbers of unrepaired DSBs are available for homology search (Shin et al. 2010; Daniel et al. 2011) and promotes SC formation independent of its role in homology search (Daniel et al. 2011). In addition, HORMAD1 is required for the efficient accumulation of ATR activity on unsynapsed chromatin in programmed DSB formation-defective Spo11 $11^{-/}$meiocytes, which are characterized by extensive asynapsis (Daniel et al. 2011). Correspondingly, HORMAD1 is required for checkpoint-mediated elimination of $S p o 11^{-/-}$oocytes during meiotic prophase. However, due to the pleiotropic effects of the Hormad1 loss-of-function mutation, the mechanistic role of HORMAD1 has remained unclear in the meiotic recruitment of ATR to unsynapsed chromatin and in meiotic prophase surveillance mechanisms. Crucially, it remained unanswered whether asynapsis is monitored during meiosis and whether inappropriate asynapsis triggers a block in prophase via an ATR-dependent surveillance mechanism that is distinct from the surveillance of DSBs.

Based on the meiotic localization of HORMAD2, we reasoned that the analysis of HORMAD2 might provide novel insights into meiotic chromosome behavior and the mechanisms of meiotic prophase checkpoints. Therefore, we examined the meiotic functions of HORMAD2 and the functional interaction of this protein with HORMAD1. Importantly, our observations strongly suggest that a distinct asynapsis surveillance mechanism exists in mammals and that asynapsis triggers elimination of meiocytes via a HORMAD2-dependent mechanism that is dispensable for the surveillance of unrepaired DSBs.

\section{Results}

Despite male infertility, no major defects in DSB metabolism and SC formation are observed in Hormad2 mutant

To address the function of HORMAD2, we disrupted the Hormad2 gene in mice (Supplemental Fig. S1). Consistent with the meiosis-specific expression of HORMAD2 (Wojtasz et al. 2009), Hormad2 ${ }^{-/}$mice are viable and do not display any obvious somatic defects. Although HORMAD2 is expressed in both oocytes and spermatocytes of wild-type mice (Wojtasz et al. 2009), female Hormad $2^{-/-}$mice are fully fertile, while males are infertile. To determine the cause of male infertility, we compared spermatogenesis on sections of wild-type and Hormad2 $2^{-/-}$testis. Hormad2 ${ }^{-/-}$spermatocytes underwent apoptosis at a stage equivalent to mid-pachytene by the end of the epithelial cycle stage IV of testis tubules (Supplemental Fig. S2).

Stage IV arrest is known to be triggered in spermatocytes that are defective in homologous SC formation between autosomes and/or in meiotic DSB repair (Barchi et al. 2005; Bolcun-Filas et al. 2007, 2009; Hamer et al. 2008a,b; Burgoyne et al. 2009). Therefore, we assessed kinetics of AE and SC formation in Hormad2 ${ }^{-/-}$meiocytes. We detected AE (STAG3, SYCP3, and HORMAD1) and SC transverse filament (SYCP1) components on nuclear spreads of meiocytes at various stages of prophase (Fig. 1A-C; Supplemental Fig. S3). AE formation and SC formation between homologous chromosomes were comparable in wild-type and Hormad $2^{-1-}$ meiocytes. Therefore, we conclude that SC formation defects are unlikely to account for the stage IV arrest that occurs in Hormad2 ${ }^{-/-}$ spermatocytes.

To test whether defects in DSB formation or repair could account for the observed stage IV arrest, we examined the behavior of recombination proteins in Hormad2 $2^{-/-}$ meiocytes (Fig. 1D-H; Supplemental Fig. S4). RAD51 and 
A

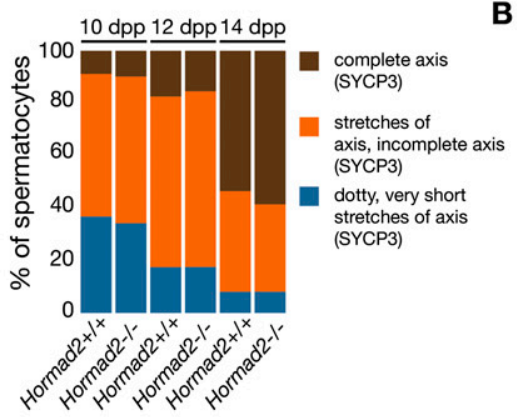

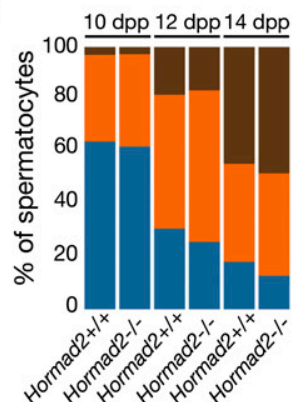

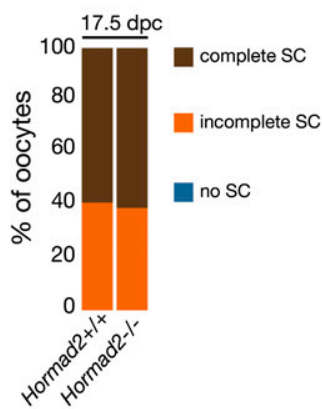

C

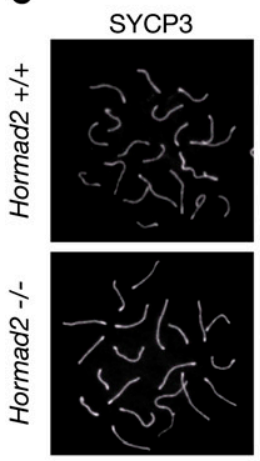

HORMAD1
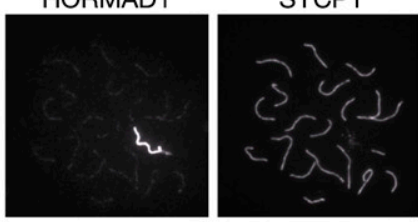

SYCP3 HORMAD
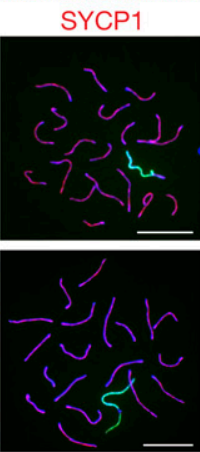

D

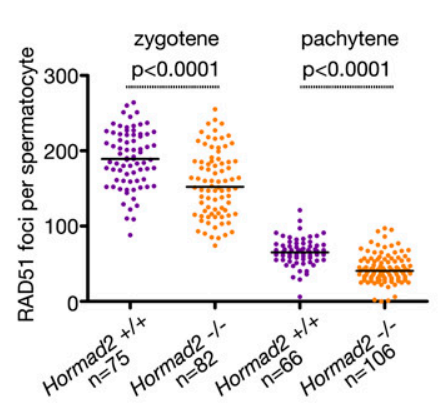

E

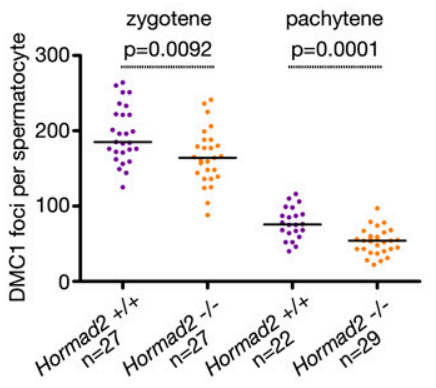

$\mathbf{F}$

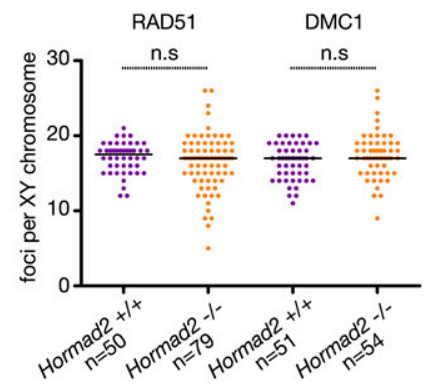

G

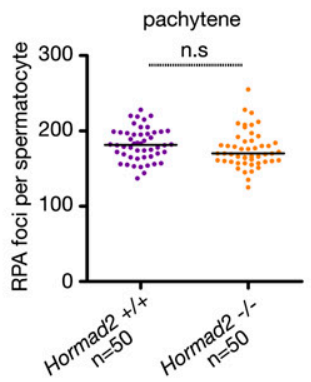

H

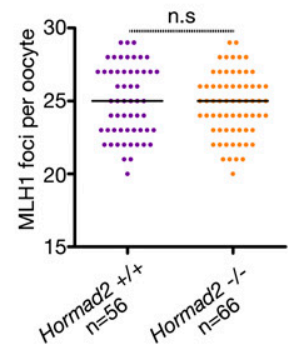

Figure 1. Chromosome axis formation, SC formation, and recombination protein foci numbers are similar in wild-type (wt) and Hormad2 ${ }^{-1-}$ meiocytes. (A) Axis development was assessed through detection of SYCP3 on nuclear spreads of spermatocytes from littermate pairs of wild-type and Hormad2 $2^{-/}$mice at the indicated ages (days post-partum, dpp). Frequencies of spermatocytes belonging to three different axis development categories are shown. (B) SC formation was assessed in spread spermatocytes from $A$ and in spread oocytes from 17.5-dpc fetuses through detection of SYCP1 (transverse filament component) by immunofluorescence (IF). Three groups of meiocytes were distinguished: cells with no SC formation (i.e., no clear SYCP1 stretches) (blue), cells with various levels of incomplete SC formation (orange) ranging from short stretches of SCs to nearly complete autosomal SCs, and complete autosomal SC formation (brown). Late pachytene wild-type spermatocytes are excluded from the graphs in $A$ and $B$. (C) Images of SYCP3, HORMAD1 (unsynapsed AEs), and SYCP1 detected by IF on nuclear spreads of early-mid-pachytene wild-type and Hormad2 ${ }^{-1-}$ spermatocytes collected from adult mice. Matched-exposure images of HORMAD1 and SYCP1 are shown. Bar, $10 \mu \mathrm{m}$. $(D-H)$ Foci numbers of early (RAD51 $[D]$ and DMC1 $[E])$, intermediate (RPA $[G])$ and late (MLH1 $[H])$ recombination proteins in zygotene and/or pachytene meiocytes and RAD51 and DMC1 foci numbers along sex chromosome axes in pachytene cells $(F)$. Median foci numbers of recombination proteins and significance of differences in foci numbers in wild-type and Hormad2 ${ }^{-1-}$ meiocytes $(P$ calculated by MannWhitney test) or lack of significance (ns) are indicated. 
DMC1 associate with resected DSB ends and promote homology search at early stages of the DSB repair process (Kolas et al. 2005; Moens et al. 2007). Following successful homology search and SC formation, RAD51 and DMC1 progressively disappear from DSB sites, and markers of intermediate stages of DSB repair, such as the ssDNAbinding protein RPA, accumulate at DSB sites (Kolas et al. 2005; Moens et al. 2007). Thus, by determining the foci numbers of RAD51, DMC1, and RPA in nuclear spreads of meiocytes, we could assess the number of DSBs and progression through DSB repair in Hormad2 ${ }^{-1-}$ meiocytes. We observed slightly lower median foci numbers of RAD51, DMC1, and RPA in Hormad2 $2^{-1-}$ spermatocytes than in wild-type spermatocytes (Fig. 1D-G; Supplemental Fig. S4A-C). Nevertheless, RAD51 and DMC1 foci numbers decreased similarly in Hormad2 ${ }^{-/-}$and wild-type meiocytes following SC formation. Correspondingly, RPA accumulated at DSB sites on synapsed AEs in Hormad2 ${ }^{-/-}$ and wild-type spermatocytes. Thus, RAD51, DMC1, and RPA behavior in the Hormad2 ${ }^{-/-}$mutant does not indicate a major role for HORMAD2 in DSB formation or DSB repair. The small reduction in the steady-state numbers of recombination foci in Hormad2 $2^{-/-}$mice may indicate that, similar to HORMAD1 (Shin et al. 2010; Daniel et al. 2011), HORMAD2 plays a role in DSB formation and/or in slowing down meiotic DSB repair kinetics, with HORMAD2 apparently being much less important than HORMAD1 in these processes.

To further assess HORMAD2's function in DSB repair, we also examined a late marker of DSB repair, MLH1, in Hormad2 ${ }^{-/-}$meiocytes. It is believed that DSB sites that are committed to becoming COs are marked by MLH1 from mid-pachytene onward in spermatocytes (Marcon and Moens 2003; Moens et al. 2007). Therefore, MLH1 foci were not expected to appear in Hormad2 ${ }^{-/-}$spermatocytes, which are eliminated in mid-pachytene. Indeed, we never observed MLH1 foci in Hormad2 ${ }^{-1-}$ spermatocytes. In contrast, Hormad2 $2^{-1-}$ females are fertile, which allowed us to examine MLH1 foci numbers in oocytes. MLH1 foci numbers and distribution were comparable in wild-type and Hormad2 ${ }^{-/-}$oocytes (Fig. $1 \mathrm{H}$; Supplemental Fig. S4D-F), which indicates that, at least in females, HORMAD2 does not have a major role in late stages of CO formation-associated DSB repair either.

\section{Interplay between HORMAD1 and HORMAD2}

Hormad1 ${ }^{-/-}$meiocytes are much more defective in DSB formation and/or repair and SC formation than Hormad2 ${ }^{-/-}$ spermatocytes. The simplest explanation for these differences is that HORMAD1, but not HORMAD2, has an important role in DSB formation/repair and SC formation. Nevertheless, it is also possible that HORMAD2 functions in these processes in wild-type meiocytes, but HORMAD1 can substitute for HORMAD2 in Hormad2 ${ }^{-/-}$ meiocytes, given that the behavior and localization pattern of HORMAD1 and HORMAD2 are very similar (Wojtasz et al. 2009). Therefore, we addressed whether these proteins interact and have overlapping functions.
To test whether HORMAD1 and HORMAD2 physically interact, we carried out immunoprecipitations with anti-HORMAD1 and HORMAD2 antibodies from testis extracts. HORMAD2 immunoprecipitations were inefficient under the tested conditions (data not shown). In contrast, anti-HORMAD1 immunoprecipitations were efficient, and HORMAD2 was detected in HORMAD1 immunoprecipitates (Fig. 2A). Therefore, we tested whether HORMAD1 and HORMAD2 interact directly by in vitro binding assays (Fig. 2B). Recombinant MBP-tagged versions of HORMAD1 and HORMAD2 expressed in bacteria showed affinity to resin-bound GST-tagged HORMAD2 and HORMAD1, respectively. This indicates that HORMAD1 and HORMAD2 may directly interact in meiocytes as well.

It is likely that HORMAD1 and HORMAD2 association with AEs is important for the meiotic functions of HORMAD1 and HORMAD2. To test whether HORMAD1 and HORMAD2 could, in principle, substitute for the functions of each other on AEs, we examined whether HORMAD1 and HORMAD2 were able to efficiently localize to AEs in Hormad2 ${ }^{-/-}$and Hormad1 ${ }^{-/-}$meiocytes, respectively. Both the pattern of HORMAD1 localization (i.e., preferential localization to unsynapsed axes) and levels of axis-associated HORMAD1 were comparable in wild-type and Hormad2 ${ }^{-1-}$ meiocytes (Fig. 1C; Supplemental Fig. S4). In contrast, chromosome axis-associated HORMAD2 levels were much lower in Hormad $1^{-1-}$ spermatocytes than in wild-type spermatocytes (Fig. 2C). Consistent with this, HORMAD2 levels were much lower in the chromatin-rich Triton X-100insoluble fraction of Hormad1 ${ }^{-1-}$ testis extracts than in the same fraction of wild-type testis extracts, while HORMAD2 levels were similar in the soluble fractions (Fig. 2D). Taken together, these results suggest that HORMAD1 is loaded onto AEs independent of HORMAD2 and that AE-bound HORMAD1 may promote HORMAD2 recruitment to AEs through direct HORMAD1-HORMAD2 interaction.

Our observations also suggest that HORMAD1 could, in principle, substitute for HORMAD2 functions on AEs, while HORMAD2 would have a limited capacity to substitute for AE-associated HORMAD1 because of low HORMAD2 levels on AEs in the Hormad1 ${ }^{-1-}$ mutant. Nevertheless, it is possible that residual HORMAD2 functionality in Hormad1 $1^{-1-}$ mutants could attenuate the DSB formation and/or repair defects and SC defects of the Hormad1 $1^{-1-}$ mutant. Consistent with this scenario, SC defects and DSB formation and/or repair defects appear to be partial in Hormad1 $1^{-1-}$ meiocytes; i.e., considerable SC formation is observed between up to onethird of homolog pairs, and steady-state numbers of single-stranded DSBs ends are reduced only approximately threefold relative to wild type (Daniel et al. 2011). To test whether HORMAD2 can partially substitute for HORMAD1 in these processes, we compared SC formation and foci numbers of recombination proteins in Hormad1 $1^{-/-}$ single mutants and Hormad1 ${ }^{-1-}$ Hormad2 $^{-1-}$ double mutants (Supplemental Fig. S5). SC formation and the numbers of recombination protein (RAD51 and RPA) foci were similar in Hormad1 ${ }^{-/-}$single-mutant and Hormad1 ${ }^{-/-}$ 


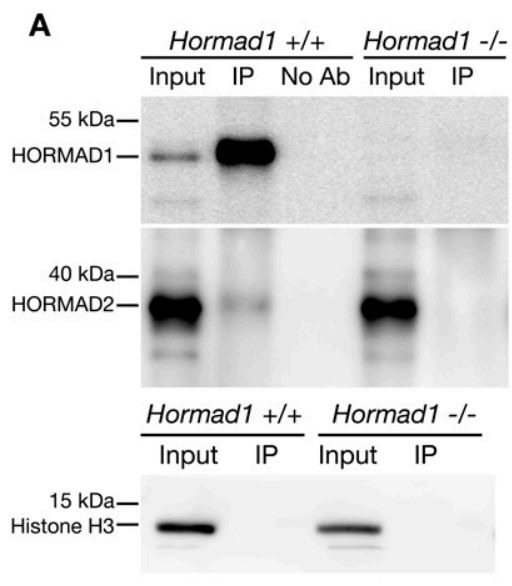

C
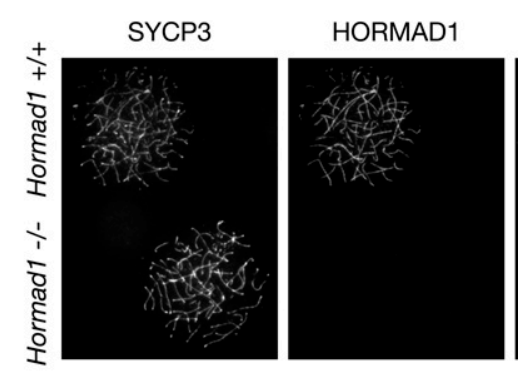

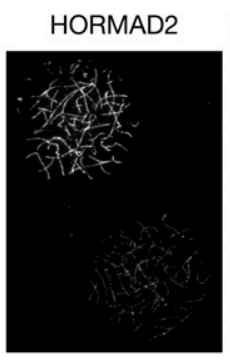

B
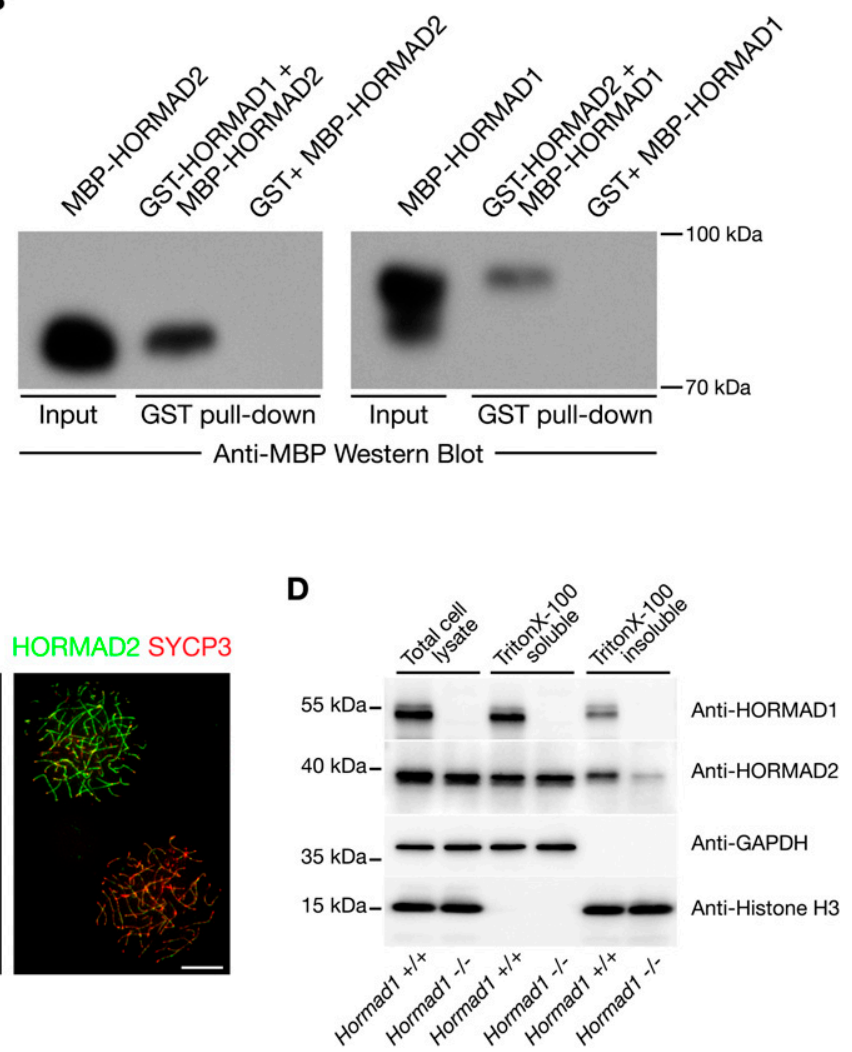

Figure 2. HORMAD1 can physically interact with HORMAD2 and permits efficient accumulation of HORMAD2 on AEs. (A) Soluble nuclear fractions of $\mathrm{Hormad1}^{+/+}$and Hormad1 ${ }^{-/-}$testes were subjected to immunoprecipitation by anti-HORMAD1 antibody. The precipitates (IP), together with input samples (equivalent to $5 \%$ of the immunoprecipitation samples) and mock immunoprecipitates (No $\mathrm{Ab}$ ), were analyzed by immunoblotting with anti-HORMAD1 (top) and anti-HORMAD2 (middle) antibodies. (Bottom) Histone H3 was detected on a separate blot in the same input and immunoprecipitation samples to test whether HORMAD1-HORMAD2 coprecipitation is mediated by chromatin. (B) Pull-down experiments with MBP- and GST-tagged versions of HORMAD1 and HORMAD2 purified from bacteria. Resin-bound GST-HORMAD1 (left) or GST-HORMAD2 (right) was used to pull down MBPHORMAD2 and MBP-HORMAD1, respectively. Pull-downs with GST protein-loaded resin were used as negative controls. Pull-down results were analyzed by immunoblotting with anti-MBP antibody. $(C)$ HORMAD1, SYCP3, and HORMAD2 were detected by IF in nuclear spreads of mixed populations of wild-type and Hormad1 ${ }^{-1-}$ spermatocytes. A zygotene wild-type cell is shown next to a zygotene stage Hormad1 ${ }^{-/-}$cell identified by HORMAD1 staining. The HORMAD2 signal is severely reduced in the Hormad1 ${ }^{-/-}$ cell relative to wild type. Bar, $10 \mu \mathrm{m}$. (D) Total, Triton X-100-soluble, and Triton X-100-insoluble extracts were prepared from testes of 12 -d-old Hormad1 $1^{+/+}$and Hormad1 ${ }^{-/-}$mice. Extracts were analyzed by immunoblotting with the indicated antibodies. Histone H3 and GAPDH were detected to control for loading.

Hormad2 $2^{-1-}$ double-mutant spermatocytes. These data suggest that either HORMAD2 does not have a major role in SC and DSB formation and/or DSB repair or HORMAD1 is required for these HORMAD2 functions and at the same time is able to substitute for HORMAD2 in these functions.

\section{Efficient meiotic sex chromosome silencing requires $H O R M A D 2$}

Why are mid-pachytene Hormad $2^{-1-}$ spermatocytes eliminated in stage IV if they show no signs of major defects in DSB formation/repair and SC formation? One possibility is that HORMAD2 is involved in the meiotic silencing of sex chromosomes, which is also called meiotic sex chromosome inactivation (MSCI). It was proposed that MSCI is essential for progression beyond mid-pachytene in sper- matocytes because there are "toxic" sex chromosomal genes whose meiotic expression is incompatible with survival of spermatocytes in stage IV testis tubules (Mahadevaiah et al. 2008; Burgoyne et al. 2009; Royo et al. 2010). MSCI is the consequence of a more general meiosisspecific phenomenon: meiotic silencing of unsynapsed chromatin (MSUC) (Turner et al. 2006). ATR associates with chromatin and unrepaired DSBs in unsynapsed chromosome regions in late zygotene/pachytene cells and phosphorylates histone H2AFX, thereby promoting MSUC. The largely nonhomologous $\mathrm{X}$ and $\mathrm{Y}$ chromosomes form SCs primarily in their short homologous pseudoautosomal regions (PAR) in spermatocytes. In early pachytene, SC can temporarily spread to nonhomologous regions of sex chromosomes; however, large parts of the X chromosome always remain unsynapsed, and upon progression to midpachytene the nonhomologous regions of both sex chro- 
mosomes are largely unsynapsed. Asynapsis of the sex chromosomes results in MSCI through the formation of a phospho-H2AFX ( $\gamma \mathrm{H} 2 \mathrm{AFX})$-rich sex chromatin domain, the sex body.

To test whether stage IV arrest occurs in Hormad2 ${ }^{-/-}$ spermatocytes due to a defect in MSUC or MSCI, we compared sex body formation in wild-type and Hormad2 ${ }^{-1-}$ spermatocytes by immunostaining $\gamma \mathrm{H} 2 \mathrm{AFX}$ (Fig. 3A). $\gamma \mathrm{H} 2 \mathrm{AFX}$-rich chromatin surrounded the unsynapsed AEs of $\mathrm{X}$ and $\mathrm{Y}$ chromosomes along their entire length in all pachytene wild-type cells $(n=523)$. Although a sex bodylike $\gamma \mathrm{H} 2 \mathrm{AFX}$-rich chromatin domain was associated with

A
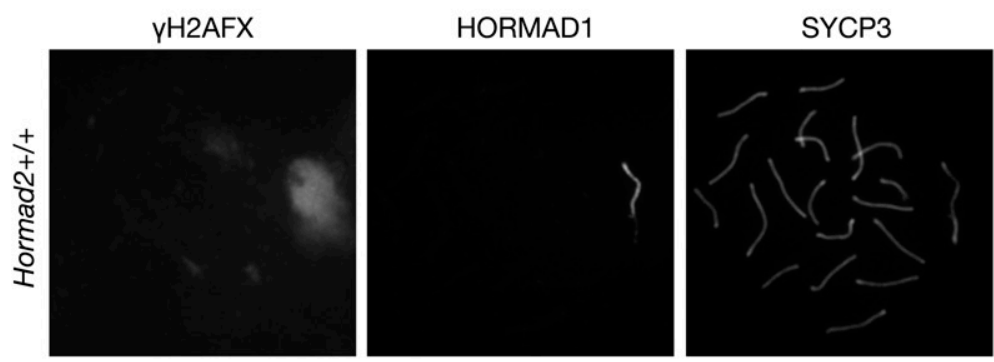

YH2AFX HORMAD1

Hormad $2+/+(n=523)$

SYCP3

Hormad2 $-/-\quad(n=580)$
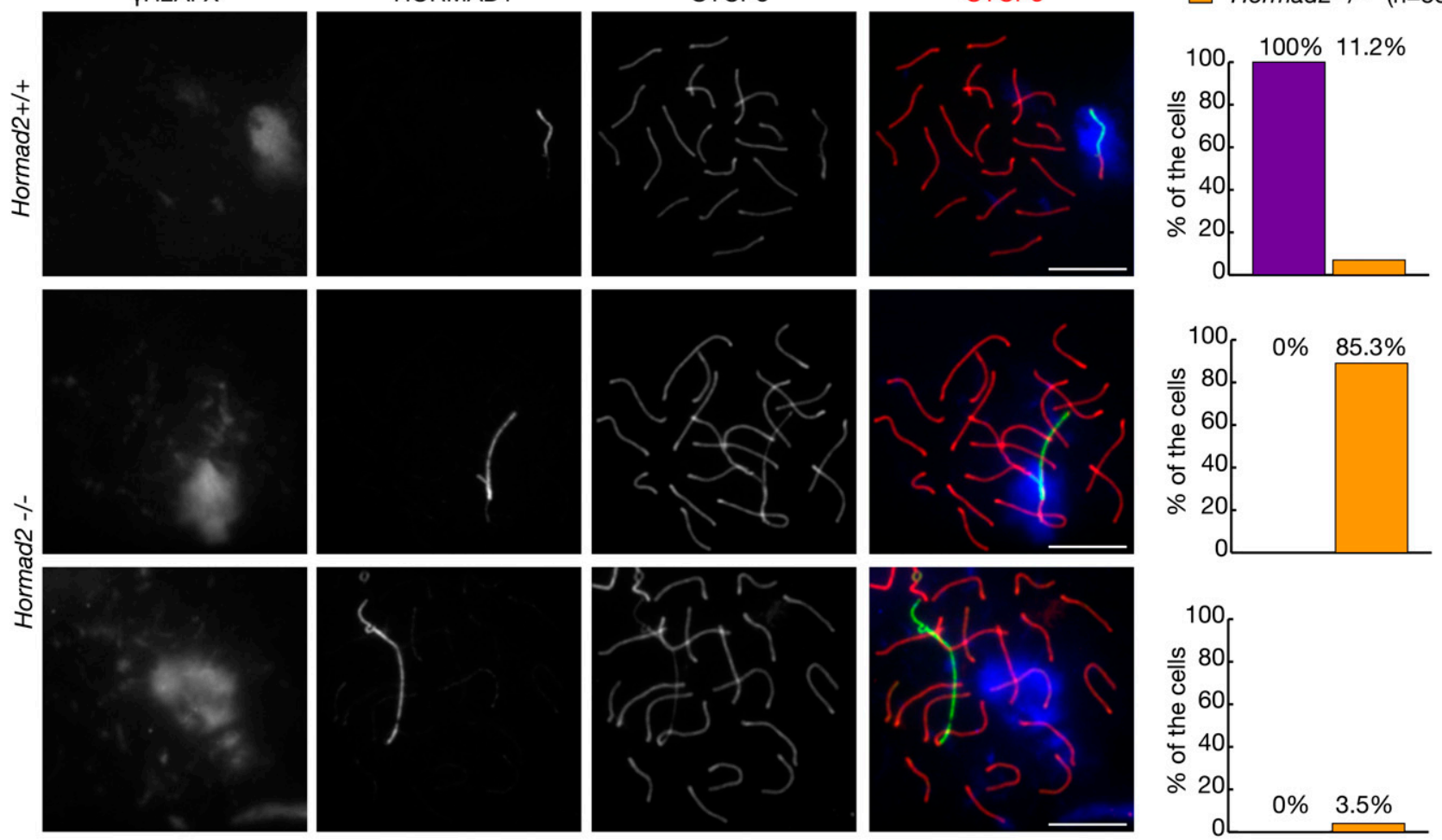

B mouse $\mathrm{X}$ chromosome
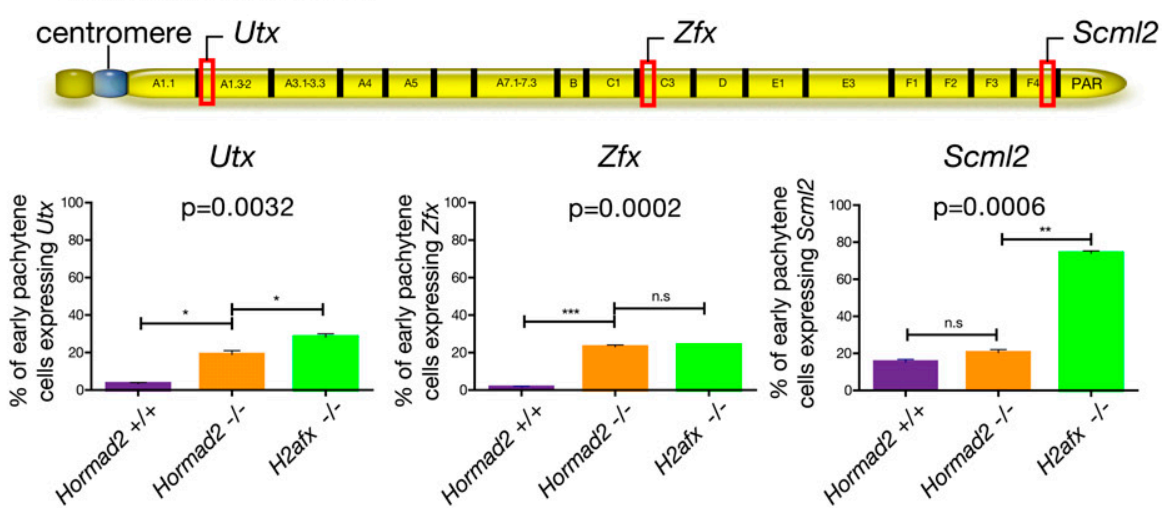

C

\begin{tabular}{|c|c|c|c|}
\hline Genotype/Probe & Utx & Zfx & Scm/2 \\
\hline Wild type & $7 / 202$ & $3 / 200$ & $31 / 202$ \\
\hline Hormad2 -/- & $38 / 200$ & $46 / 200$ & $51 / 201$ \\
\hline H2afx -/- & $54 / 201$ & $48 / 200$ & $149 / 201$ \\
\hline
\end{tabular}

Figure 3. Sex body formation is defective in Hormad2 ${ }^{-/-}$spermatocytes. (A) $\gamma \mathrm{H} 2 \mathrm{AFX}$, HORMAD1, and SYCP3 were detected in nuclear spreads of wild-type and Hormad2 $2^{-/-}$pachytene spermatocytes collected from 21-d-old mice. Matched-exposure images of $\gamma \mathrm{H} 2 \mathrm{AFX}$ are shown. Three different types of cells were distinguished: $\gamma \mathrm{H} 2 \mathrm{AFX}$-rich chromatin completely (top row, wild type) or partially (middle row, Hormad2 ${ }^{-1}$ ) surrounds the HORMAD1 marked unsynapsed sex chromosome axes, or the $\gamma \mathrm{H} 2 \mathrm{AFX}-\mathrm{rich}$ chromatin domain does not overlap with the sex chromosome axes (bottom row, Hormad2 ${ }^{-/}$). The frequency of these categories in wild-type and Hormad2 ${ }^{-1-}$ spermatocytes is shown on the right. Bar, $10 \mu \mathrm{m}$. (B) Frequency of wild-type, Hormad2 ${ }^{-1-}$, and $H 2 a f x^{-/-}$ early pachytene spermatocytes expressing $U t x, Z f_{X}$, and $S c m l 2$ as measured by RNA FISH. Significance was determined using one-way ANOVA $\left(P\right.$-value shown) and Tukey's multiple comparison test; $\left.\left(^{\star}\right) P<0.05 ;\left(^{\star \star}\right) P<0.01 ;\left.\right|^{\star \star \star}\right) P<0.001$. (Top) Schematic of mouse $\mathrm{X}$ chromosome shows locations of $U t x, Z f_{X}$, and $S c m l 2$. $(C)$ Table of raw values from $B$. The number of early pachytene spermatocytes expressing $U t x, Z f_{X}$, or Scml2 out of the total number of cells is shown. 
$\mathrm{X}$ and $\mathrm{Y}$ chromosomes in the vast majority of pachytene Hormad2 ${ }^{-1-}$ spermatocytes $(96.5 \%, n=580)$, various proportions of $\mathrm{X}$ or $\mathrm{Y}$ AEs did not overlap with the $\gamma \mathrm{H} 2 \mathrm{AFX}$-rich chromatin in $85.3 \%$ of the cells (Fig. 3A; Supplemental Fig. S6A). In $3.5 \%$ of Hormad2 ${ }^{-1-}$ spermatocytes, the $\gamma \mathrm{H} 2 \mathrm{AFX}$-rich chromatin domain did not form or did not overlap at all with sex chromosome AEs. $\gamma \mathrm{H} 2 \mathrm{AFX}$ accumulation along the X-chromosome axis was nonuniform in Hormad2 $2^{-1-}$ spermatocytes. $\gamma \mathrm{H} 2 \mathrm{AFX}$ tended to accumulate on sex chromatin in the vicinity of the PAR region $(73 \%, n=182)$, while the centromeric one-third of the $\mathrm{X}$ chromosome, which is on the opposite end of the X chromosome (Fig. 3B), lacked or had reduced levels of $\gamma \mathrm{H} 2 \mathrm{AFX}$ in most Hormad2 ${ }^{-1-}$ spermatocytes $(68 \%, n=182)$. Other markers of sex body formation, such as an anti-XLR recognized meiotic antigen (Calenda et al. 1994; Reynard et al. 2007) and MDC1 (Ichijima et al. 2011), also show reduced localization to sex chromosomes, mirroring the abnormal localization of $\gamma \mathrm{H} 2 \mathrm{AFX}$ on sex chromatin (Supplemental Fig. S6B-D). This indicates that sex body formation is incomplete and spatially limited in Hormad2 $2^{-1-}$ spermatocytes.

In addition to these sex body abnormalities, the frequency of sex chromosome pairing defects was elevated in Hormad2 ${ }^{-1-}$ spermatocytes (data not shown). During early-mid-pachytene, $\mathrm{X}$ and $\mathrm{Y}$ chromosomes remained unsynapsed in $11 \%(n=400)$ of Hormad $^{-/-}$spermatocytes as opposed to $0.5 \%(n=400)$ of wild-type spermatocytes. In addition, one end of $\mathrm{X}$ and/or $\mathrm{Y}$ chromosomes associated with the end of a fully synapsed autosome in $19 \%$ of Hormad2 ${ }^{-1-}$ spermatocytes $(n=200)$. Given that two unrelated mutant mouse lines, $\mathrm{H} 2 \mathrm{afx}_{\mathrm{X}}^{-/-}$and $M d c 1^{-1-}$, that are defective in $\gamma \mathrm{H} 2 \mathrm{AFX}$ accumulation on sex chromosomes display similar sex chromosome pairing defects (Fernandez-Capetillo et al. 2003; Ichijima et al. 2011), it is likely that these defects are a consequence of defective sex body formation and/or $\gamma \mathrm{H} 2 \mathrm{AFX}$ accumulation on sex chromosomes in Hormad2 $^{-/-}$ spermatocytes.

To test whether abnormal $\gamma \mathrm{H} 2 \mathrm{AFX}$ accumulation on $\mathrm{X}$ and $\mathrm{Y}$ chromosomes affected MSCI, we used RNA FISH to assess transcriptional activity of three X-linked genes $S c m l 2, Z f_{X}$, and $U t x$, which are located in the proximity of the PAR region and in the middle and at the centromeric end of the X chromosome, respectively (Fig. 3B,C; Supplemental Fig. S6E). We compared transcription of these genes in wild-type, Hormad2 ${ }^{-1-}$, and $\mathrm{H}_{2} \mathrm{afx}_{\mathrm{X}}{ }^{-1-}$ early pachytene spermatocytes. $\mathrm{H}_{2} a \mathrm{f}_{\mathrm{x}}{ }^{-1-}$ spermatocytes served as a positive control for loss of MSCI (Fernandez-Capetillo et al. 2003). Frequencies of pachytene spermatocytes with gene expression from $Z f_{X}$ and Utx genes were significantly elevated in both Hormad2 ${ }^{-/-}$ and $H 2 a \mathrm{fx}^{-1-}$ mice relative to wild-type mice. In contrast to $Z f_{X}$ and $U t x$, the PAR-proximal $S c m l 2$ gene was inefficiently silenced in wild-type spermatocytes, possibly due to the proximity of synapsed and transcriptionally active regions or occasional spreading of SC to the Scml2 locus. The frequency of cells with gene expression from $5 \mathrm{cml} 2$ was not elevated significantly in Hormad2 ${ }^{-1-}$ mice but was strongly elevated in $\mathrm{H}_{2} \mathrm{af}_{\mathrm{x}}{ }^{-/-}$mice relative to wild-type mice. These gene expression measurements correspond well with the asymmetric $\gamma \mathrm{H} 2 \mathrm{AFX}$ accumulation pattern on sex chromatin in pachytene Hormad2 ${ }^{-/-}$ spermatocytes and indicate failure of MSCI, particularly in X-chromosome regions that are distant from the PAR region.

\section{HORMAD2 is required for ATR activity recruitment to unsynapsed axes}

What is the primary reason for MSCI failure in Hormad2 ${ }^{-/-}$ spermatocytes? RAD51 and DMC1 foci numbers are comparable on sex chromosomes of Hormad2 ${ }^{-1-}$ and wild-type spermatocytes (Fig. 1F). Thus, a reduction in DSB numbers is unlikely to be the cause of MSCI failure in the Hormad2 $2^{-1-}$ spermatocytes. To address whether MSCI failure in Hormad2 $2^{-1-}$ spermatocytes is caused by a defect in ATR activity recruitment to unsynapsed chromosomes, we examined the localization of ATR and ATR activators/modulators (BRCA1 and TOPBP1) in nuclear spreads of Hormad2 ${ }^{-/}$spermatocytes (Fig. 4; Supplemental Fig. S7). In wild-type spermatocytes, ATR, BRCA1, and TOPBP1 accumulate to high levels along the unsynapsed sex chromosome AEs (Keegan et al. 1996; Plug et al. 1998; Moens et al. 1999; Perera et al. 2004; Turner et al. 2004, 2005). At lower density, ATR and TOPBP1 also localize to chromatin all over the $\gamma \mathrm{H} 2 \mathrm{AFX}$ rich sex body (Fig. 4A; Supplemental Fig. S7A). This chromosome-wide spreading of ATR and TOPBP1 is believed to depend on MDC1 that colocalizes with $\gamma \mathrm{H} 2 \mathrm{AFX}$ in the sex body (Supplemental Fig. S6D; Ichijima et al. 2011). Cloud-like ATR and TOPBP1 staining was still observed in $\gamma \mathrm{H} 2 \mathrm{AFX}$-rich chromatin domains in Hormad2 $2^{-1-}$ spermatocytes (Fig. 4A; Supplemental Fig. S7A), which was consistent with the observation that MDC1 colocalized with $\gamma \mathrm{H} 2 \mathrm{AFX}$ in both normal and abnormal sex bodies in wild-type and Hormad2 ${ }^{-1-}$ spermatocytes, respectively (Supplemental Fig. S6D). In contrast, ATR, BRCA1, and TOPBP1 failed to accumulate strongly along the unsynapsed sex chromosome axes (Fig. 4; Supplemental Fig. S7A). Nevertheless, we observed weak punctate staining of ATR, BRCA1, and TOPBP1 on the unsynapsed axes of sex chromosomes. This raised the possibility that Hormad2 $2^{-/-}$spermatocytes were primarily defective in recruitment of ATR to unsynapsed chromosome axes but were proficient in ATR activity recruitment to unrepaired DSBs. To test this possibility, we compared the localization of DMC1 with ATR and TOPBP1 on the X-chromosome axis of Hormad2 ${ }^{-/-}$ spermatocytes (Fig. 4C; Supplemental Fig. S7B). We found that $92 \%(n=20$ cells $)$ and $90 \%(n=21$ cells $)$ of DMC1 foci on X chromosomes were associated with ATR and TOPBP1 foci, respectively. Consistent with this, we found that $\gamma \mathrm{H} 2 \mathrm{AFX}$-rich chromatin along the $\mathrm{X}$-chromosome axis was associated with regions that contained ATR, BRCA1, TOPBP1, or DMC1 foci (Fig. 4A,B; Supplemental Fig. S7A,C). Thus, ATR activity appeared to be recruited to DSB sites on the unsynapsed X-chromosome axis in pachytene Hormad2 ${ }^{-/-}$ spermatocytes. 
A
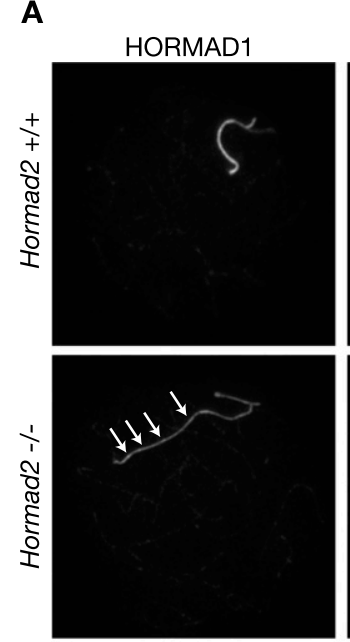

B
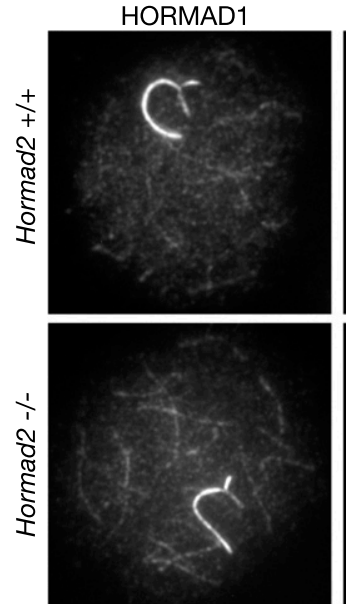

C

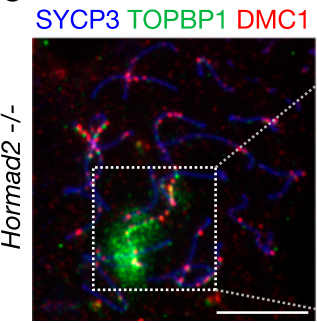

YH2AFX
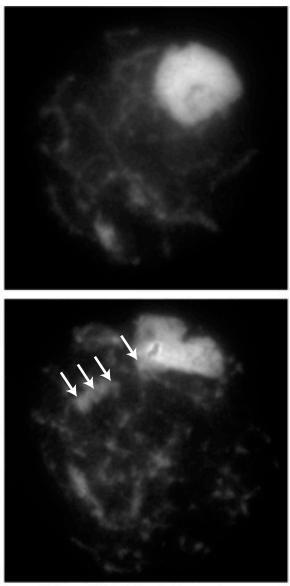

YH2AFX
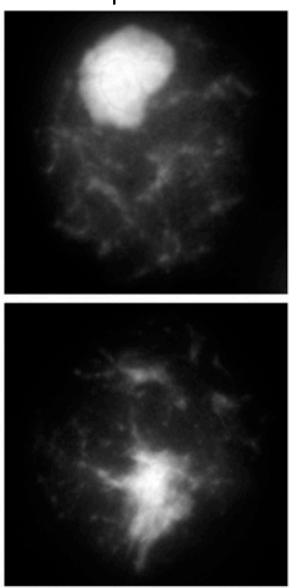

SYCP3

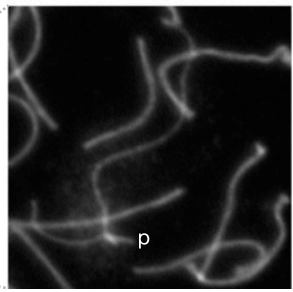

ATR low
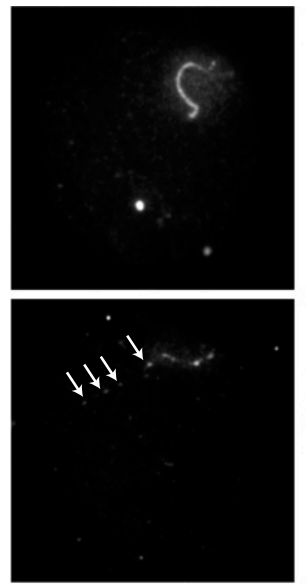

BRCA1 low
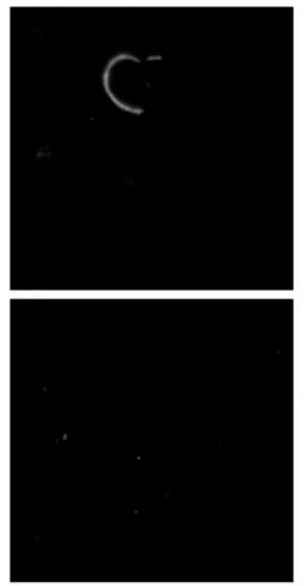

TOPBP1

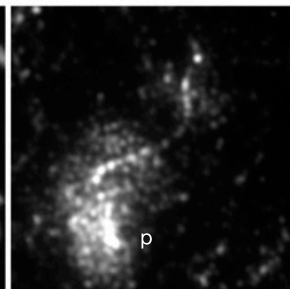

ATR high
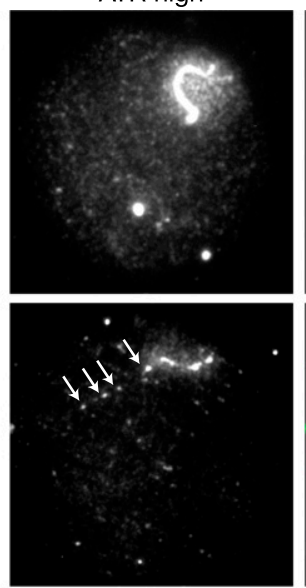

BRCA1 high
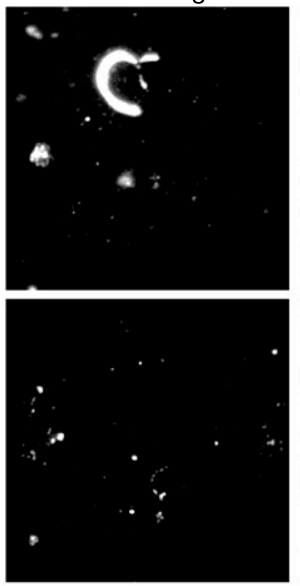

DMC1

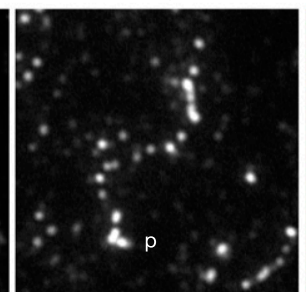

YH2AFX ATR HORMAD1
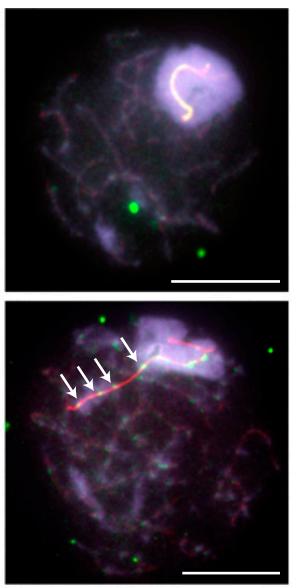

үH2AFX BRCA1
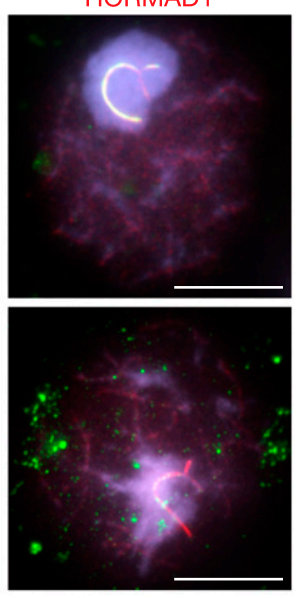

TOPBP1 DMC1

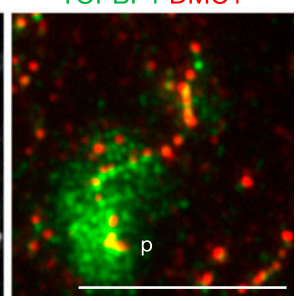

Figure 4. ATR, BRCA1, and TOPBP1 levels on unsynapsed chromosome axes are low in Hormad2 ${ }^{-/-}$spermatocytes. Indicated proteins were detected in nuclear spreads of wild-type and Hormad2 ${ }^{-/-}$early pachytene spermatocytes. Matched-exposure images of ATR and BRCA1 are shown in wild-type and Hormad2 ${ }^{-1-}$ meiocytes in $A$ and $B$, respectively. ATR and BRCA1 staining is much lower on $\mathrm{X}$ and $\mathrm{Y}$ chromosome axes in the Hormad2 ${ }^{-1-}$ spermatocytes than in the wild-type spermatocytes. Nevertheless, a cloud-like staining of ATR is observed in $\gamma \mathrm{H} 2 \mathrm{AFX}$-rich chromatin in both the wild-type and mutant spermatocyte. High-exposure images of ATR ([A] ATR high) and BRCA1 ([B] BRCA1 high) are shown to highlight residual punctate staining along chromosome axes in Hormad2 ${ }^{-/}$ spermatocytes. Note the local accumulation of $\gamma \mathrm{H} 2 \mathrm{AFX}$ (arrows) in the vicinity of ATR foci along the X-chromosome axis. (C) Zoomedin image of the inset shows sex chromosomes in a Hormad2 ${ }^{-/-}$spermatocyte. Note the punctate accumulation of TOPBP1 along the sex chromosome axes in the vicinity of DMC1 foci. (p) PAR region in C. Bars, $10 \mu \mathrm{m}$.

To further test the hypothesis that HORMAD2 is specifically needed for ATR activity recruitment to unsynapsed AEs, we examined the effect of Hormad2 ${ }^{-/-}$ mutation in meiocytes that are defective in either DSB and SC formation $\left(S p o 11^{-/-}\right)$(Baudat et al. 2000; Romanienko and Camerini-Otero 2000) or DSB repair and
SC formation (Dmc1 $1^{-/-}$) (Pittman et al. 1998). Spo11 ${ }^{-/-}$ meiocytes do not form programmed meiotic DSBs, hence homology search and SC formation between homologs fail (Baudat et al. 2000; Romanienko and Camerini-Otero 2000). Nevertheless, extensive SC formation is observed between nonhomologous chromosomes. During prophase 
stages equivalent to late zygotene and pachytene, $\gamma \mathrm{H} 2 \mathrm{AFX}-$ rich chromatin domains form surrounding a subset of unsynapsed chromosome axes in a substantial fraction of Spo11 $11^{-1-}$ meiocytes $163 \%$ of spermatocytes in adult males, $n=250 ; 59 \%$ of oocytes in newborn females, $n=200$ ). These $\gamma \mathrm{H} 2 \mathrm{AFX}$-rich chromatin domains are called pseudosex bodies because they rarely overlap with sex chromosomes (Barchi et al. 2005; Bellani et al. 2005). It is assumed that pseudo-sex bodies form in Spo11 ${ }^{-1-}$ meiocytes because ATR is recruited to unsynapsed axes (Mahadevaiah et al. 2008) and subsequently spreads to chromatin loops with the help of MDC1 (Ichijima et al. 2011). If HORMAD2 was needed for efficient ATR recruitment to unsynapsed AEs independent of programmed DSBs, we would expect that efficient pseudo-sex body formation required HORMAD2. Total $\gamma \mathrm{H} 2 \mathrm{AFX}$ levels and the frequency of pseudo-sex body formation were strongly reduced in Spo11 ${ }^{-/-}$Hormad2 ${ }^{-/-}$meiocytes relative to Spo11-1meiocytes (Fig. 5A-D; Supplemental Fig. S8A). Although ATR always colocalized with $\gamma \mathrm{H} 2 \mathrm{AFX}$ within pseudosex bodies in both Spo11 ${ }^{-/-}$and Spo11 ${ }^{-/-}$Hormad2 $^{-/-}$ spermatocytes, strong ATR recruitment to unsynapsed AEs within pseudo-sex bodies was observed in $11 \%$ of Spo11 ${ }^{-1-}$ spermatocytes $(n=200)$ and was never observed in the Spo11 $11^{-1-}$ Hormad2 $2^{-/-}$spermatocytes that formed pseudo-sex bodies $(n=200)$ (Fig. 5A). Thus, efficient pseudo-sex body formation and efficient recruitment of ATR to unsynapsed AEs and chromatin require HORMAD2 in the DSB formation-defective Spo11 1 - background. These defects are unlikely to be caused by altered meiotic progression or altered SC formation, since spermatocytes are eliminated at stage IV,

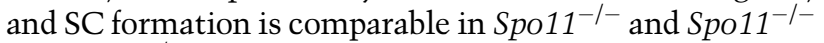
Hormad2 ${ }^{-/-}$meiocytes (data not shown).

In contrast to Spo $11^{-/-}$meiocytes, $D m c 1^{-/-}$meiocytes efficiently form DSBs (Pittman et al. 1998), but DSB repair is believed to be defective at the strand invasion step. Consequently, homology search and homologous SC formation fail in $D \mathrm{mc1}^{-/-}$meiocytes (Pittman et al. 1998; Yoshida et al. 1998). Unlike in Spo11 $11^{-/-}$meiocytes, pseudo-sex bodies do not form in $\mathrm{Dmc1}^{-/-}$meiocytes, probably because recruitment of ATR to large numbers of unrepaired DSBs distributed all over the genome does not allow the concentration of ATR and other DDR components into a defined chromatin domain (Barchi et al. 2005). Consistent with this idea, $\gamma \mathrm{H} 2 \mathrm{AFX}$ accumulates on chromatin all over the nucleus of $D m c 1^{-/-}$spermatocytes at a stage that is equivalent to zygotene or pachytene. If HORMAD2 was not required for recruitment of ATR to unrepaired DSBs during meiosis, we would expect colocalization of unrepaired DSB markers (e.g., RPA, and ATR) and also accumulation of $\gamma \mathrm{H} 2 \mathrm{AFX}$ on chromatin of $\mathrm{Dmc1}^{-1-}$ Hormad2 $^{-/-}$spermatocytes. Indeed, 96\% of RPA foci were associated with ATR in $D m c 1^{-/-}$ Hormad2 ${ }^{-1-}$ spermatocytes $(n=15$ cells), which is comparable with the $97 \%$ association observed in $D m c 1^{-1-}$ control cells ( $n=15$ cells) (Fig. 5E; Supplemental Fig. S8B). $\gamma \mathrm{H} 2 \mathrm{AFX}$ also accumulates on chromatin across the nucleus of all the zygotene-pachytene Dmc1 ${ }^{-1-}$ Hormad2 $^{-1-}$ meiocytes (Fig. 5F; Supplemental Fig. S8C). The simplest in- terpretation of these experiments is that HORMAD2 is primarily required for efficient recruitment of ATR activity to unsynapsed axes and has little or no role in ATR activity recruitment to unrepaired DSBs in either sex.

To address whether HORMAD2 could, in principle, promote recruitment of ATR activity to unsynapsed axes via physical interactions with ATR or ATR activators, we immunoprecipitated soluble fractions of HORMAD1, HORMAD2, and ATR. Under the tested conditions, these immunoprecipitation experiments did not indicate that ATR, TOPBP1, or BRCA1 form stable soluble complexes with HORMAD1 or HORMAD2 (data not shown).

\section{HORMAD2 is required for a prophase checkpoint that is presumably activated by asynapsis}

SC and/or DSB repair defects result in elimination of oocytes at the end of the first meiotic prophase in perinatal mice (Di Giacomo et al. 2005). It was proposed that perinatal elimination of defective oocytes is triggered by either MSUC-mediated silencing of a random set of essential genes or persistent ATR activity and ATR signaling during late prophase (Burgoyne et al. 2009). Given that HORMAD2 is required for efficient accumulation of ATR activity on unsynapsed chromatin in both sexes, we reasoned that HORMAD2 might be required for meiotic prophase checkpoints in females.

To test this possibility, we sectioned through ovaries of 6-wk-old wild-type, Hormad2 ${ }^{-/-}, S_{\text {Spo11 }}^{-/-}, \mathrm{Spo11}^{-/-}$ Hormad2 ${ }^{-/-}, \mathrm{Dmc1}^{-/-}$, and Dmc1 ${ }^{-/-}$Hormad2 $^{-/-}$mice and determined the number of oocytes in every eighth section (Fig. 6A; Supplemental Fig. S9). Oocyte numbers were comparable in wild-type, Hormad2 ${ }^{-1-}$, and $S p o 11^{-1-}$ Hormad $2^{-/-}$females. In contrast, oocyte numbers were much lower in Spo11-/- ovaries, and oocytes were completely absent from $\mathrm{Dmc1}^{-/-}$and $\mathrm{Dmc1}^{-1-} \mathrm{Hormad}^{-/-}$ ovaries. Thus, the elimination of $S p o 11^{-/-}$oocytes required HORMAD2, while elimination of $D \mathrm{mc1}^{-/-}$oocytes did not. These results correspond well with the observation that ATR is efficiently recruited to unrepaired DSBs and that $\gamma \mathrm{H} 2 \mathrm{AFX}$ efficiently accumulates on chromatin across the nucleus in $D \mathrm{mc1}^{-/-}$Hormad2 ${ }^{-1-}$ meiocytes. Taken together, our observations suggest that HORMAD2 is required for a checkpoint mechanism in females that is activated by persistent asynapsis.

\section{HORMAD2 is needed to prevent production of first meiotic metaphase oocytes with defects in chiasmata formation}

HORMAD2 is not required for female fertility, as the litter sizes of wild-type (average litter size: 5.47 pups, $n=6$ females, 21 litters) and Hormad2 ${ }^{-1-}$ (average litter size: 5.71 pups, $n=6$ females, 21 litters) littermate females are similar. This raises the question of how important the HORMAD2-dependent female prophase checkpoint mechanism is in wild-type mice. It was reported that homolog pairing and/or SC formation are delayed or defective on one or more chromosomes in up to $10 \%-15 \%$ of oocytes in wild-type fetuses at 17.5 or $18.5 \mathrm{~d}$ post-coitum (dpc), which is a stage when most oocytes are in mid-pachytene stage 
A
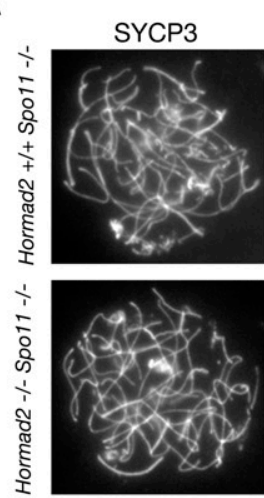

YH2AFX
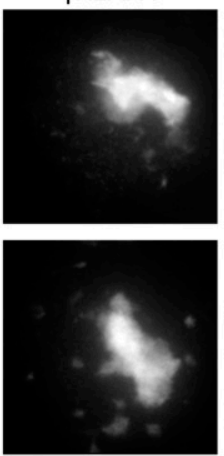

ATR
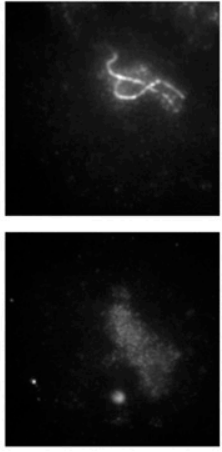

YH2AFX SYCP3
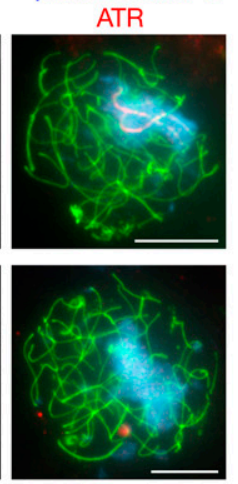

B

- Hormad2 +/+ Spo11 -/- $(\mathrm{n}=250)$

- Hormad2 -/- Spo11 -/- $\quad(n=250)$

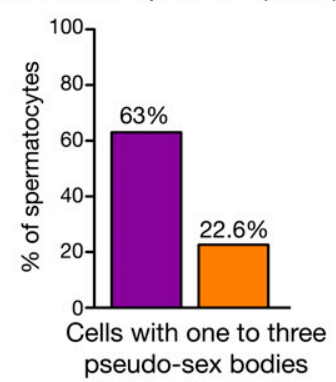

E
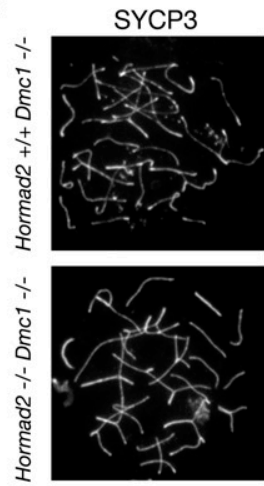

F
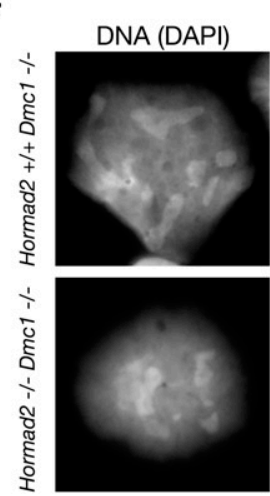

C

- Hormad $2+/+$ Spo11 -/- $(n=200)$

- Hormad2 -/- Spo11 -/- $\quad(n=200)$
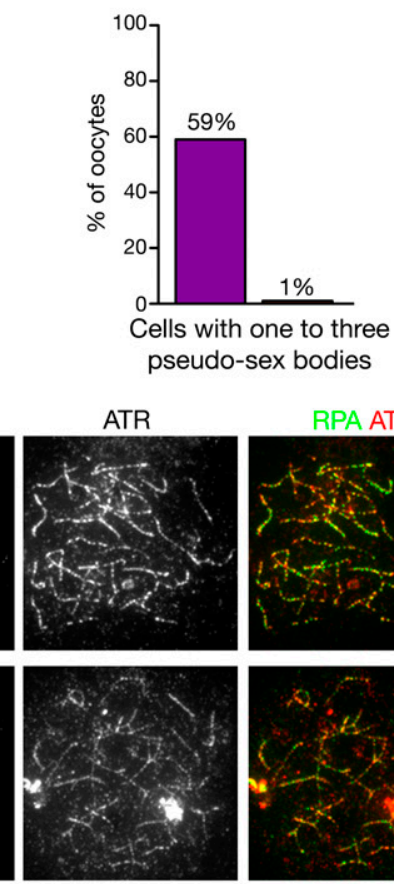

SYCP3
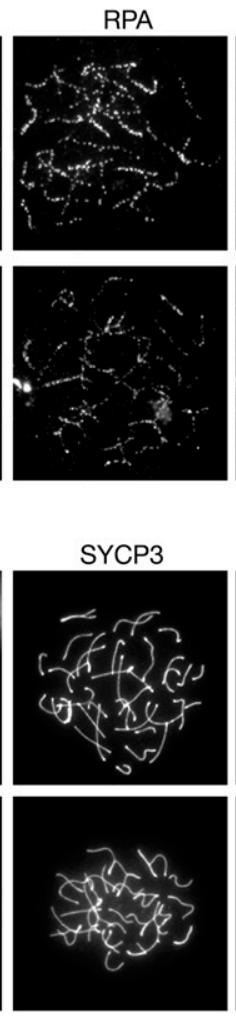

YH2AFX
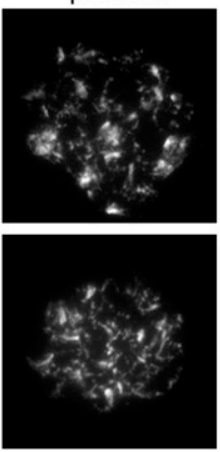

RPA ATR
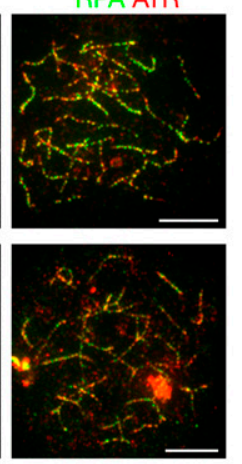

DNA SYCP3 YH2AFX
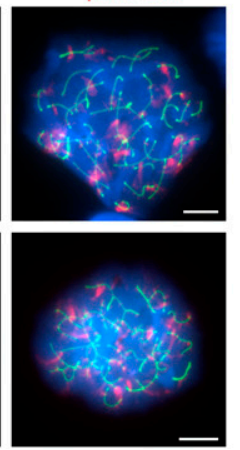

Figure 5. Accumulation of ATR activity on unsynapsed chromatin in Spo11 ${ }^{-/-}$meiocytes is defective in the absence of HORMAD2. $(A, E, F)$ Nuclear spreads of spermatocytes of the indicated genotypes. Spermatocytes are shown with fully formed chromosome axes; i.e., at a stage corresponding to late zygotene or early pachytene in wild-type spermatocytes. Matched-exposure images of ATR and $\gamma \mathrm{H} 2 \mathrm{AFX}$ are shown. $(A)$ While in a fraction $(11 \%)$ of $\mathrm{Hormad2}^{+/+}$Spo11 $1^{-1-}$ spermatocytes ATR accumulates on chromosome axes within $\gamma \mathrm{H} 2 \mathrm{AFX}$-rich pseudo-sex bodies (top panel), in Hormad2 ${ }^{-/}$Spo11 ${ }^{-/-}$spermatocytes (bottom panel), ATR accumulates only as a cloud in the small fraction $(22.6 \%)$ of spermatocytes that form pseudo-sex bodies. $(B, C)$ The frequency of occurrence of pseudo-sex bodies in spermatocytes $(B$; collected from 8-wk-old mice) and oocytes $(C$; collected from newborn mice) with full-length chromosome axes. $(D)$ Quantification of $\gamma \mathrm{H} 2 \mathrm{AFX}$ signal in spermatocytes with full-length chromosome axes (collected from adult mice) shows a significant

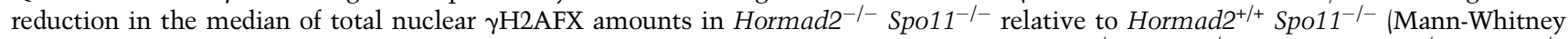
test). (E) ATR foci are associated with axes in the vicinity of RPA foci in both Hormad2 ${ }^{+/}$Dmc1 $1^{-/-}$and Hormad2 $2^{-/-}$Dmc1 ${ }^{-/-}$ spermatocytes. $(F)$ HORMAD2 is not required for accumulation of $\gamma \mathrm{H} 2 \mathrm{AFX}$ on chromatin in $\mathrm{Dmc1} 1^{-/-}$spermatocytes. Bars, $10 \mu \mathrm{m}$. 
A

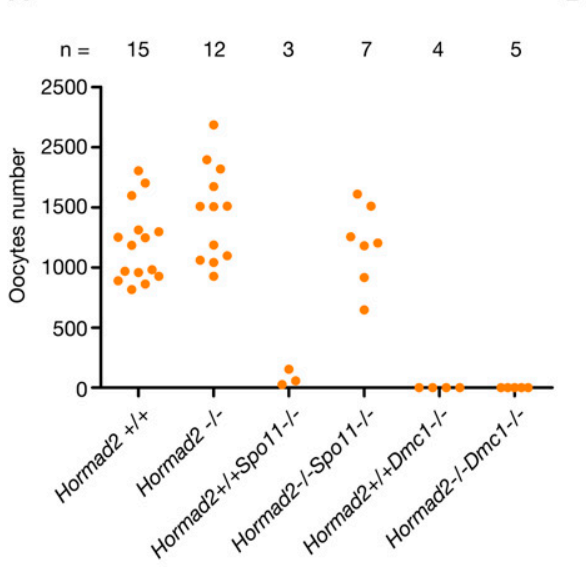

B
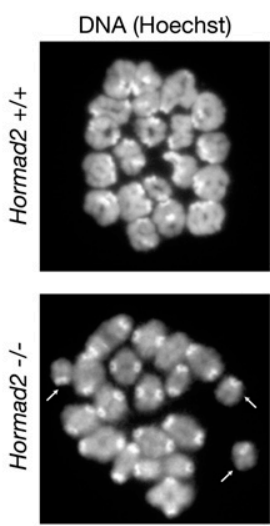
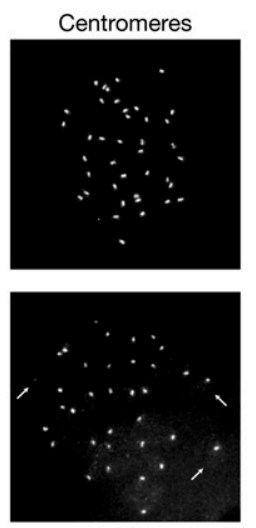

DNA Centromeres
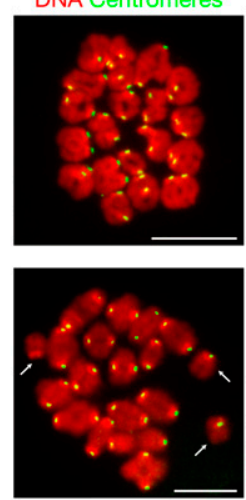

C

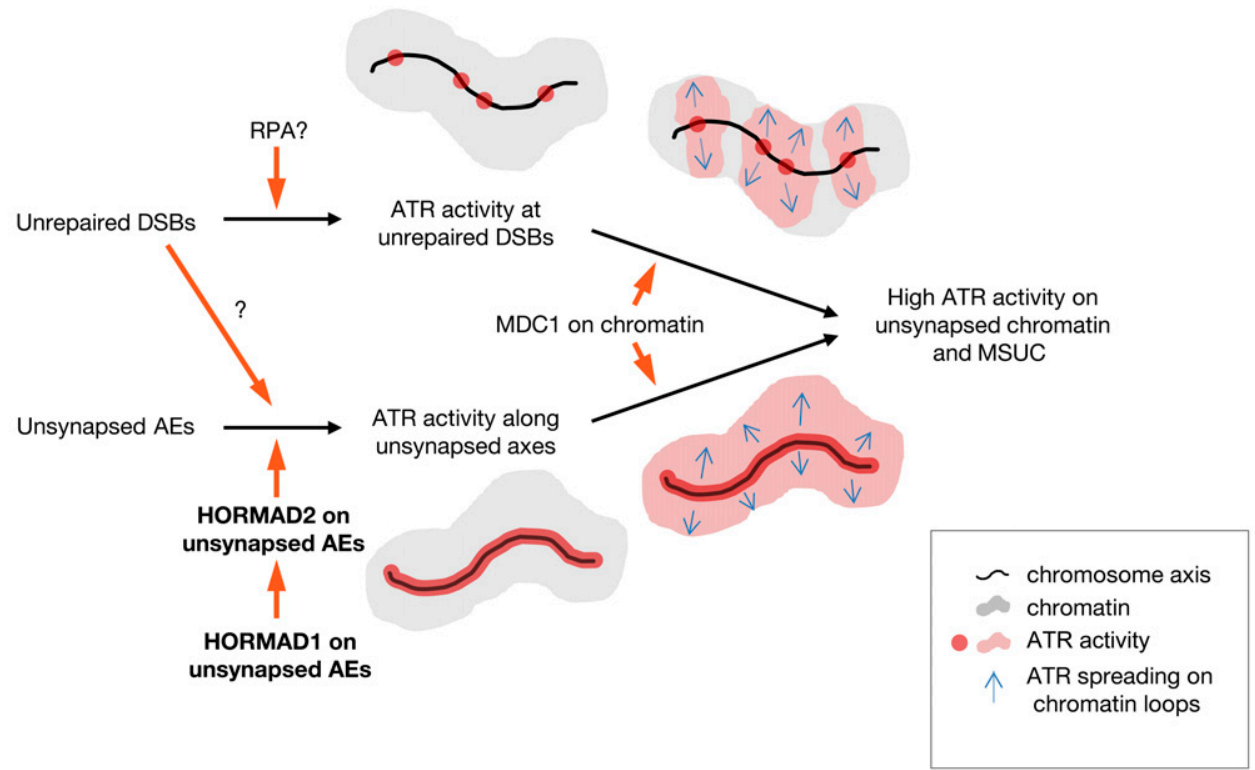

Figure 6. HORMAD2 is required for a prophase checkpoint that eliminates SC formation-defective Spo11 ${ }^{-1-}$ oocytes. (A) Sum of oocyte numbers on every eighth section of sectioned-through ovary pairs in 6-wk-old mice of indicated genotypes. Each data point represents a mouse. $(B)$ Images of chromosome spreads of in vitro matured wild-type and Hormad2 ${ }^{-1-}$ oocytes at the first meiotic metaphase. DNA and centromeres were detected by Hoechst and IF, respectively. Chiasmata connect all pairs of homologs in the wildtype oocytes. In contrast, the Hormad2 $2^{-1-}$ oocyte contain several univalents (arrows). Univalents scatter over a larger area during nuclear spreading in the Hormad2 ${ }^{-/-}$oocyte; therefore, it was not possible to include all 40 chromosomes in the image. Bars, $10 \mu \mathrm{m}$. (C) Model for the recruitment of ATR activity to unsynapsed chromatin in pachytene meiocytes. Orange arrows and black arrows represent activation promotion and processes, respectively. We propose that ATR activity is recruited to unrepaired DSBs along unsynapsed chromosome axes by a mechanism that is not dependent on HORMAD2. Although ATR activity at DSBs can promote spreading of ATR activity to chromatin loops adjacent to DSBs with the help of MDC1 and $\gamma \mathrm{H} 2 \mathrm{AFX}$, it cannot ensure spreading of ATR activity to all of the unsynapsed chromatin. Efficient sex body formation and spreading of ATR activity to chromatin loops that are not associated with unrepaired DSBs require HORMAD1- and HORMAD2-dependent recruitment of ATR activity along the unsynapsed chromosome axes. This process may be facilitated by DSBs through DSB-dependent phosphorylation of HORMAD1 and HORMAD2.

(Alton et al. 2008; Kouznetsova et al. 2009). It is likely that these SC defects either become corrected or trigger elimination of oocytes. It is also conceivable that homolog pairing defects and/or SC defects may result in $\mathrm{CO}$ formation defects in affected oocytes. Therefore, if SCdefective oocytes succeed in maturation to the first meiotic metaphase, they may contain univalents; i.e., unpaired homologs that lack chiasmata. As a corollary, we may expect elevated numbers of defective first meiotic metaphase oocytes that contain univalents in
Hormad2 $2^{-1-}$ mice if HORMAD2 was important for the elimination of SC-defective oocytes. To test this hypothesis, we determined the frequency of SC defects in pachytene oocytes, which were identified by the presence of the pachytene marker MLH1 recombination protein, in wildtype and Hormad2 ${ }^{-1-}$ 17.5-dpc fetuses (for an example, see Supplemental Fig. S3C) and determined the frequency of in vitro matured metaphase I oocytes that contained univalents in wild-type and Hormad2 $2^{-/}$adult mice (for an example, see Fig. 6B). While the frequency of SC-defective 
pachytene oocytes was very similar in wild-type fetuses (12 out of 154 cells, two fetuses) and Hormad2 ${ }^{-1-}$ fetuses (12 out of 162, two fetuses), the frequency of univalentcontaining metaphase I oocytes showed a small but clear increase $(P=0.0268$, two-tailed Fisher exact test $)$ in the Hormad $2^{-/-}$mice (10 out of 242 oocytes, $4.13 \%, 15$ mice) relative to wild-type mice ( 1 out of 194 oocytes, $0.52 \%, 14$ mice). Although we cannot exclude that this increase in chiasmata formation defects in Hormad2 $2^{-1-}$ mice is caused by a small defect in SC formation or in DSB repair that was not detected by our analysis of Hormad2 ${ }^{-/-}$ oocytes, these data are consistent with the hypothesis that a HORMAD2-dependent meiotic prophase checkpoint safeguards the quality of the female germline by eliminating SC-defective oocytes in wild-type mice.

\section{Discussion}

It has been hypothesized that quality control of $\mathrm{CO}$ formation-related processes during meiosis involves the monitoring of both DSB repair defects and asynapsis (Burgoyne et al. 2009). Current models evoke important roles for ATR activation and recruitment to unrepaired DSBs and/or unsynapsed chromatin in meiotic quality control of $\mathrm{CO}$ formation-related processes in both sexes (Burgoyne et al. 2009). In spermatocytes, DSB repair defects and asynapsis in excess of normal XY chromosome asynapsis are proposed to trigger apoptosis through titrating ATR activity away from the sex chromosomes, thereby causing MSCI failure. In oocytes, persistent ATR activity at unrepaired DSBs and/or on unsynapsed chromatin is believed to cause apoptosis. Nevertheless, it has been difficult to determine the relative importance of different lesions in triggering ATR-dependent prophase checkpoint responses because pairing defects and asynapsis are linked to delayed or defective DSB repair.

In some mutant mice (e.g., in $D \mathrm{mc}^{-/-}$and Trip $13^{-/-}$ mice), DSB repair defects are considered to be the likely primary cause of meiocyte apoptosis (Pittman et al. 1998; Di Giacomo et al. 2005; Li and Schimenti 2007; Burgoyne et al. 2009; Roig et al. 2010). In fact, DSB repair defects may trigger meiocyte elimination during prophase in Trip $13^{-l-}$ cells in the absence of major SC formation defects (Li and Schimenti 2007; Roig et al. 2010). Nevertheless, DSB repair defects are unlikely to be the only lesions that are monitored by meiotic surveillance mechanisms. Spo $11^{-1-}$ mutants are defective in programmed meiotic DSB formation (Baudat et al. 2000; Romanienko and Camerini-Otero 2000). Despite the absence or strongly reduced numbers of DSBs, Spo11 $11^{-/}$meiocytes are eliminated at stage IV in males and perinatally in females (Barchi et al. 2005; Di Giacomo et al. 2005). Given that ATR activity accumulates on unsynapsed chromatin and that $S p o 11^{-I-}$ meiocytes are characterized by extensive asynapsis, it seems likely that incomplete $\mathrm{SC}$ formation is detected by ATR-dependent meiotic surveillance mechanisms, leading to the elimination of $S p o 11^{-/-}$meiocytes in both sexes.

It has been hypothesized that ATR-promoted checkpoint mechanisms, including MSUC and MSCI, rely on two essential consecutive steps: asynapsis detection coupled with MDC1-independent recruitment of ATR activity to unsynapsed AEs, which is followed by the MDC1dependent spreading of ATR activity from unsynapsed AEs to associated chromatin loops (Ichijima et al. 2011). Although this model postulates a crucial role for ATR activity recruitment to unsynapsed AEs, the existence and importance of a presumed asynapsis surveillance mechanism remained questionable in the absence of identified proteins that are specifically required for the elimination of SC-defective meiocytes and for the meiosis-specific accumulation of ATR and ATR-activating DDR proteins (BRCA1 and TOPBP1) on unsynapsed AEs and/or associated chromatin loops.

Our data suggest that the primary role of HORMAD2 is to ensure the recruitment of ATR and ATR activators to unsynapsed AEs. HORMAD2 is not required for DSB formation/repair, SC formation, or the accumulation of ATR activity on chromatin loops in the vicinity of unrepaired DSBs. Crucially, defective accumulation of ATR, BRCA1, and TOPBP1 along the unsynapsed sex chromosome AEs is coupled with defective MSCI in Hormad2 ${ }^{-1-}$ spermatocytes and inefficient elimination of SC-defective Hormad $2^{-1-}$ oocytes. Taken together, these observations provide compelling support for the hypothesis that HORMAD2-promoted accumulation of DDR components along the unsynapsed AEs is crucial for efficient MSCI in spermatocytes and the elimination of asynaptic oocytes.

Consistent with the hypothesis that either inappropriate MSUC or persistent ATR activity triggers elimination of SC formation-defective oocytes perinatally, HORMAD2 is required for efficient pseudo-sex body formation in

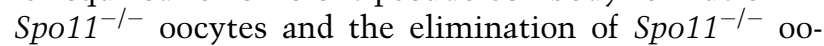
cytes. In contrast, HORMAD2 is not required for the recruitment of ATR to unrepaired DSBs, the accumulation of ATR activity on chromatin in $D m \mathrm{mi}^{-1-}$ meiocytes, and the elimination of $D \mathrm{mc1}^{-1-}$ oocytes. Given that HORMAD2 is depleted from AEs in response to SC formation, these observations strongly suggest that HORMAD2 is a sensor component of a prophase surveillance mechanism that monitors asynapsis. Thus, our observations provide clear evidence for the existence of a long-debated asynapsis surveillance mechanism that is distinct from surveillance mechanisms that monitor DSBs in mammals. In addition, the observation that the number of in vitro matured oocytes with chiasmata errors is elevated in Hormad2 $2^{-1-}$ mice relative to wild-type mice is consistent with the idea that such a HORMAD2dependent asynapsis surveillance mechanism eliminates oocytes with synaptic errors in wild-type backgrounds, thereby safeguarding the quality of the germline. Considering that meiotic recombination errors are a major cause of the generation of aneuploid human embryos (Hassold et al. 2007), the discovery and future analysis of the HORMAD2-dependent asynapsis surveillance mechanism will likely have an impact on our understanding of human aneuploidies.

Since both HORMAD1 and HORMAD2 are required for the elimination of Spo11 ${ }^{-/-}$oocytes and for pseudosex body formation, these two proteins appear to have 
nonoverlapping functions in the surveillance of asynapsis. HORMAD2 association to AEs is strongly reduced in the absence of HORMAD1, and HORMAD1 and HORMAD2 directly interact in vitro. Therefore, it is conceivable that HORMAD1 performs its essential function in asynapsis surveillance and ATR activity recruitment to unsynapsed chromatin by recruiting HORMAD2 to unsynapsed AEs. In turn, axis-bound HORMAD2 may promote recruitment of ATR and/or ATR activators to unsynapsed AEs. Immunoprecipitation experiments of HORMAD1 and HORMAD2 have not indicated the existence of stable soluble complexes between HORMAD1 or HORMAD2 and DDR proteins. Provided that HORMAD2 promotes recruitment of ATR activity to unsynapsed axes by physically interacting with DDR components, these observations may indicate that only the insoluble axis-bound form of HORMAD2 can bind and activate DDR proteins efficiently. Interestingly, another HORMA domain protein, the spindle assembly checkpoint protein MAD2, is known to adopt two different conformations (De Antoni et al. 2005). When microtubules are not attached stably to kinetochores, MAD2 associates with kinetochores and adopts a conformation that allows MAD2 to bind and inhibit the anaphasepromoting complex component CDC20, which results in a metaphase arrest. It is tempting to speculate that a conformation change that activates checkpoint functions in response to monitored lesions is a conserved paradigm among HORMA domain proteins with checkpoint sensor functions. Thus, it will be important to establish whether association of HORMAD1 and HORMAD2 with unsynapsed chromosome axes induces changes in the conformations of HORMAD1 and HORMAD2 that facilitate physical interaction with DDR proteins.

Taken together, our data suggest a comprehensive model for the coordination of CO formation-associated events and progression through meiotic prophase. We propose that following the formation of AEs and DSBs, two alternative mechanisms initiate accumulation of ATR activity on chromatin in unsynapsed regions (Fig. 6C). ATR is recruited to unrepaired meiotic DSBs independent of HORMAD2, possibly through a mechanism that is similar to the RPA-dependent recruitment of ATR to singlestranded DSB ends in mitotic cells (Zou and Elledge 2003). At the same time, ATR and ATR activators are recruited to unsynapsed AEs through a HORMAD1- and HORMAD2-dependent process. Following recruitment of ATR activity to unrepaired DSBs and/or unsynapsed AEs, ATR activity spreads to chromatin loops with the help of MDC1 (Ichijima et al. 2011). This last step ensures efficient MSUC and/or MSCI and amplification of ATR signaling in response to unrepaired DSBs and asynapsis.

Although surveillance of unrepaired DSBs and asynapsis relies on distinct mechanisms, there might be crosstalk between these mechanisms. Despite accumulation of ATR on chromatin in $100 \%$ of pseudo-sex bodies in Spo11 $11^{-/}$spermatocytes, we observed clear ATR enrichment along unsynapsed chromosome axes within pseudosex bodies in only $11 \%$ of pseudo-sex body-containing cells. In contrast, ATR enrichment was observed along unsynapsed sex chromosome axes in all wild-type earlymid-pachytene spermatocytes. These observations indicate that ATR accumulation on unsynapsed chromosome axes is not as efficient in the DSB formation-deficient Spo11 $11^{-/-}$spermatocytes as in the DSB formation-proficient wild-type spermatocytes. Curiously, both HORMAD1 and HORMAD2 are phospho-proteins. HORMAD1 and HORMAD2 phosphorylation is reduced in $S p o 11^{-/-}$ testes (Fukuda et al. 2012; V Boonsanay and A Tóth, unpubl.). Thus, it is enticing to hypothesize that formation of DSBs promotes the phosphorylation of HORMAD1 and HORMAD2 and that DSB-induced phosphorylation enhances the ability of HORMAD1 and HORMAD2 to recruit ATR activity along unsynapsed axes. Such a mechanism may amplify checkpoint signaling from DSBs, thereby ensuring that small numbers of unrepaired DSBs can trigger oocyte apoptosis. It may also ensure that DSBs whose repair was delayed but not blocked could trigger elimination of oocytes in late prophase even after all DSBs were repaired, provided that such a delayed DSB repair associated with persistent asynapsis. DSB-induced activation of HORMAD1 and HORMAD2 could also ensure that the asynapsis surveillance mechanism does not become reactivated and that ATR activity is not recruited to desynapsing AEs in DSB repair-proficient wild-type diplotene cells despite the reassociation of low levels of HORMAD1 and HORMAD2 with desynapsing AEs (Wojtasz et al. 2009).

Meiotic HORMA domain proteins have been also implicated in the coordination and quality control of meiotic prophase processes in nonmammalian organisms such as Caenorhabditis elegans and budding yeast (Couteau and Zetka 2005; Martinez-Perez and Villeneuve 2005; Carballo et al. 2008; MacQueen and Hochwagen 2011). Nevertheless, meiotic prophase quality-control mechanisms and the functions of HORMA domain proteins appear to show considerable divergence between various taxa. For example, in budding yeast, DSB formation-defective and SC formation-defective spo11 mutant cells do not arrest in meiotic prophase, which may be interpreted as a sign of budding yeast lacking an asynapsis surveillance mechanism (Hochwagen and Amon 2006). Curiously, the budding yeast meiotic HORMA domain protein Hop 1 is required for the prophase arrest of meiotic cells that fail to repair DSBs and to complete SC formation in meiosis (Carballo et al. 2008). Consequently, Hop1 was proposed to be required for an efficient prophase checkpoint response to unrepaired DSBs. Thus, HORMA domain proteins may be involved in the surveillance of two distinct processes, asynapsis and unrepaired DSBs, in mammals and budding yeast, respectively. Nevertheless, it cannot be excluded that a Hop1-mediated asynapsis surveillance mechanism that depends on Spo11-induced DSBs exists (Hochwagen and Amon 2006) and that this asynapsis surveillance mechanism is a crucial component of the meiotic prophase checkpoint in budding yeast. Such a scenario would be consistent with the observation that the Hopl-mediated checkpoint response requires phosphorylation of Hop1 by the budding yeast orthologs 
of the mammalian ATM and ATR DDR kinases (Carballo et al. 2008). Thus, asynapsis surveillance may be an evolutionarily conserved feature of meiotic quality control and a core function of meiotic HORMA domain proteins, but HORMA domain protein-mediated surveillance of asynapsis may depend on SPO11-induced DSBs and on DSB-dependent phosphorylation of HORMA domain proteins to various extents in different taxa.

To summarize, our data provide compelling evidence for the existence of a meiosis-specific surveillance mechanism that monitors inappropriate asynapsis during meiotic prophase and safeguards the quality of the mammalian germline. Our data suggest that two meiotic HORMA domain proteins, HORMAD1 and HORMAD2, adapt the DDR sensor kinase ATR for the meiosis-specific task of sensing asynapsis by promoting the recruitment of ATR and/or ATR activators to unsynapsed chromosome axes. Future work will need to identify the physical interactions between chromosome axis components and HORMA domain and DDR proteins in order to unravel the molecular mechanisms underlying asynapsis surveillance in mammals.

\section{Materials and methods}

\section{Targeting of Hormad2 and animal experiments}

The Hormad2 targeting construct was designed according to a multipurpose allele strategy (Supplemental Fig. S1; Testa et al. 2004). We generated mice that carried either of two distinct Hormad2-null alleles: Hormad2insertion and Hormad2 ${ }^{\text {deletion }}$ (Supplemental Fig. S1). The phenotypes of these alleles regarding SC formation, DSB formation/repair, abnormal sex body formation in spermatocytes, and rescue of oocytes in a Spo11-1background are essentially indistinguishable. Therefore, in the text, we do not distinguish between the Hormad2 ${ }^{\text {insertion }}$ and Hormad2deletion alleles and refer to both of them as the Hormad2 $2^{-}$allele. All of our conclusions are based on experiments that were performed at least twice in Hormad2 deletion mice. Mice carrying Hormad1-, Dmc1-, or Spo11-null alleles were described earlier (Pittman et al. 1998; Baudat et al. 2000; Daniel et al. 2011). Experiments were performed in a mixed background.

\section{Immunofluorescence (IF)}

Preparation and immunostaining of testis-ovary cryosections and nuclear surface spreads of meiocytes and the antibodies used for IF were described previously (Wojtasz et al. 2009; Daniel et al. 2011). RNA FISH in combination with IF staining was carried out as described before (Mahadevaiah et al. 2009). To produce metaphase I oocytes for nuclear spreading, oocytes were collected from antral follicles of adult (>12-wk-old) mice and cultured in $\mathrm{M} 2$ medium for $6 \mathrm{~h}$ at $37.5^{\circ} \mathrm{C}$. Staging of meiocytes, quantification of $\gamma \mathrm{H} 2 \mathrm{AFX}$ signal in spread meiocytes, and oocyte numbers were performed as described before except that oocyte numbers were counted in every eighth section of sectioned ovaries (Wojtasz et al. 2009; Daniel et al. 2011).

\section{Extracts, immunoprecipitations, and pull-down experiments}

Total testis extracts were prepared by boiling finely chopped testis in Laemmli buffer for $5 \mathrm{~min}$. To prepare Triton X-100soluble and -insoluble testis extract fractions, we incubated testis cell suspensions of 12- or 13-d-post-partum (dpp) mice in a Triton X-100 lysis buffer (50 mM Tris at $\mathrm{pH} 7.5,150 \mathrm{mM} \mathrm{NaCl}$, $0.25 \%$ Triton X-100, protease and phosphatase inhibitors) for $30 \mathrm{~min}$ at $4^{\circ} \mathrm{C}$ and separated soluble and insoluble fractions by centrifugation at 20,000 relative centrifugal force (rcf) for 10 min. Immunoprecipitations were carried out following standard protocols in Brij-58-soluble nuclear testis extracts (details in the Supplemental Material). Pull-down experiments were performed as before with minor modifications (see the Supplemental Material) (Remenyi et al. 2005).

\section{Acknowledgments}

We thank A. Hientzsch for technical assistance; R. Jessberger for sharing ideas, antibodies (anti-SYCP3 and anti-STAG3), and advice; S. Namekawa for anti-BRCA1 antibody; J. Chen for the anti-TOPBP1 antibody; E. Marcon for the anti-RPA antibody; and M.A. Handel for anti-H1t antibody. We are grateful to D. Knapp for revising the manuscript. The Deutsche Forschungsgemeinschaft (grants TO421/4-1 SPP1384, TO421/3-1, and TO421/3-2) and the Sächsisches Staatsministerium für Wissenschaft und Kunst supported L.W., M.B., K.D., and A.T.; the Medical Research Council supported J.M.A.T. and, together with the Marshall Scholarship Programme, supported J.M.C.; A.R. is a János Bolyai Fellow of the Hungarian Academy of Sciences and a Wellcome Trust International Senior Research Fellow (081665/ $\mathrm{Z} / 06 / \mathrm{Z})$; the DFG research center and cluster of excellence to the Center for Regenerative Therapies Dresden (CRTD) supported the work of K.A.; and an EFRE grant (Europäischer Fonds für Regionale Entwicklung) supported K.A., J.F., and A.F.S.

\section{References}

Alton M, Lau MP, Villemure M, Taketo T. 2008. The behavior of the X-and Y-chromosomes in the oocyte during meiotic prophase in the B6. $\mathrm{Y}^{\mathrm{TIR}}$ sex-reversed mouse ovary. Reproduction 135: 241-252.

Barchi M, Mahadevaiah S, Di Giacomo M, Baudat F, de Rooij DG, Burgoyne PS, Jasin M, Keeney S. 2005. Surveillance of different recombination defects in mouse spermatocytes yields distinct responses despite elimination at an identical developmental stage. Mol Cell Biol 25: 7203-7215.

Baudat F, de Massy B. 2007. Regulating double-stranded DNA break repair towards crossover or non-crossover during mammalian meiosis. Chromosome Res 15: 565-577.

Baudat F, Manova K, Yuen JP, Jasin M, Keeney S. 2000. Chromosome synapsis defects and sexually dimorphic meiotic progression in mice lacking Spo11. Mol Cell 6: 989-998.

Bellani MA, Romanienko PJ, Cairatti DA, Camerini-Otero RD. 2005. SPO11 is required for sex-body formation, and Spo11 heterozygosity rescues the prophase arrest of $\mathrm{Atm}^{-1-}$ spermatocytes. J Cell Sci 118: 3233-3245.

Bolcun-Filas E, Costa Y, Speed R, Taggart M, Benavente R, De Rooij DG, Cooke HJ. 2007. SYCE2 is required for synaptonemal complex assembly, double strand break repair, and homologous recombination. J Cell Biol 176: 741-747.

Bolcun-Filas E, Hall E, Speed R, Taggart M, Grey C, de Massy B, Benavente R, Cooke HJ. 2009. Mutation of the mouse Syce1 gene disrupts synapsis and suggests a link between synaptonemal complex structural components and DNA repair. PLoS Genet 5: e1000393. doi: 10.1371/journal.pgen.1000393.

Burgoyne PS, Mahadevaiah SK, Turner JM. 2009. The consequences of asynapsis for mammalian meiosis. Nat Rev Genet 10: $207-216$.

Calenda A, Allenet B, Escalier D, Bach JF, Garchon HJ. 1994. The meiosis-specific Xmr gene product is homologous to the 
lymphocyte Xlr protein and is a component of the XY body. EMBO J 13: 100-109.

Carballo JA, Johnson AL, Sedgwick SG, Cha RS. 2008. Phosphorylation of the axial element protein Hop1 by Mec1/Tel1 ensures meiotic interhomolog recombination. Cell 132: 758 770.

Couteau F, Zetka M. 2005. HTP-1 coordinates synaptonemal complex assembly with homolog alignment during meiosis in C. elegans. Genes Dev 19: 2744-2756.

Daniel K, Lange J, Hached K, Fu J, Anastassiadis K, Roig I, Cooke HJ, Stewart AF, Wassmann K, Jasin M, et al. 2011. Meiotic homologue alignment and its quality surveillance are controlled by mouse HORMAD1. Nat Cell Biol 13: 599-610.

De Antoni A, Pearson CG, Cimini D, Canman JC, Sala V, Nezi L, Mapelli M, Sironi L, Faretta M, Salmon ED, et al. 2005. The Mad1/Mad2 complex as a template for Mad2 activation in the spindle assembly checkpoint. Curr Biol 15: 214-225.

Di Giacomo M, Barchi M, Baudat F, Edelmann W, Keeney S, Jasin M. 2005. Distinct DNA-damage-dependent and -independent responses drive the loss of oocytes in recombination-defective mouse mutants. Proc Natl Acad Sci 102: 737742 .

Fernandez-Capetillo O, Mahadevaiah SK, Celeste A, Romanienko PJ, Camerini-Otero RD, Bonner WM, Manova K, Burgoyne P, Nussenzweig A. 2003. H2AX is required for chromatin remodeling and inactivation of sex chromosomes in male mouse meiosis. Dev Cell 4: 497-508.

Fukuda T, Daniel K, Woitasz L, Toth A, Hoog C. 2010. A novel mammalian HORMA domain-containing protein, HORMAD1, preferentially associates with unsynapsed meiotic chromosomes. Exp Cell Res 316: 158-171.

Fukuda T, Pratto F, Schimenti JC, Turner JM, Camerini-Otero RD, Hoog C. 2012. Phosphorylation of chromosome core components may serve as axis marks for the status of chromosomal events during mammalian meiosis. PLOS Genet 8: e1002485. doi: 10.1371/journal.pgen.1002485.

Hamer G, Novak I, Kouznetsova A, Hoog C. 2008a. Disruption of pairing and synapsis of chromosomes causes stage-specific apoptosis of male meiotic cells. Theriogenology 69: 333-339.

Hamer G, Wang H, Bolcun-Filas E, Cooke HI, Benavente R, Hoog C. 2008b. Progression of meiotic recombination requires structural maturation of the central element of the synaptonemal complex. J Cell Sci 121: 2445-2451.

Hassold T, Hall H, Hunt P. 2007. The origin of human aneuploidy: Where we have been, where we are going. Hum Mol Genet 16: R203-R208. doi: 10.1093/hmg/ddm243.

Hochwagen A, Amon A. 2006. Checking your breaks: Surveillance mechanisms of meiotic recombination. Curr Biol 16: R217-R228. doi: 10.1016/j.cub.2006.03.009.

Ichijima Y, Ichijima M, Lou Z, Nussenzweig A, Camerini-Otero RD, Chen J, Andreassen PR, Namekawa SH. 2011. MDC1 directs chromosome-wide silencing of the sex chromosomes in male germ cells. Genes Dev 25: 959-971.

Keegan KS, Holtzman DA, Plug AW, Christenson ER, Brainerd EE, Flaggs G, Bentley NJ, Taylor EM, Meyn MS, Moss SB, et al. 1996. The Atr and Atm protein kinases associate with different sites along meiotically pairing chromosomes. Genes Dev 10: 2423-2437.

Keeney S, Giroux CN, Kleckner N. 1997. Meiosis-specific DNA double-strand breaks are catalyzed by Spo11, a member of a widely conserved protein family. Cell 88: 375-384.

Kolas NK, Marcon E, Crackower MA, Hoog C, Penninger JM, Spyropoulos B, Moens PB. 2005. Mutant meiotic chromosome core components in mice can cause apparent sexual dimorphic endpoints at prophase or X-Y defective malespecific sterility. Chromosoma 114: 92-102.
Kouznetsova A, Wang $\mathrm{H}$, Bellani $\mathrm{M}$, Camerini-Otero RD, Jessberger R, Hoog C. 2009. BRCA1-mediated chromatin silencing is limited to oocytes with a small number of asynapsed chromosomes. J Cell Sci 122: 2446-2452.

Li XC, Schimenti JC. 2007. Mouse pachytene checkpoint 2 (trip13) is required for completing meiotic recombination but not synapsis. PLoS Genet 3: e130. doi: 10.1371/journal.pgen. 0030130.

MacQueen AJ, Hochwagen A. 2011. Checkpoint mechanisms: The puppet masters of meiotic prophase. Trends Cell Biol 21: 393-400.

Mahadevaiah SK, Bourc'his D, de Rooij DG, Bestor TH, Turner JM, Burgoyne PS. 2008. Extensive meiotic asynapsis in mice antagonises meiotic silencing of unsynapsed chromatin and consequently disrupts meiotic sex chromosome inactivation. J Cell Biol 182: 263-276.

Mahadevaiah SK, Costa Y, Turner JM. 2009. Using RNA FISH to study gene expression during mammalian meiosis. Methods Mol Biol 558: 433-444.

Marcon E, Moens P. 2003. MLH1p and MLH3p localize to precociously induced chiasmata of okadaic-acid-treated mouse spermatocytes. Genetics 165: 2283-2287.

Martinez-Perez E, Villeneuve AM. 2005. HTP-1-dependent constraints coordinate homolog pairing and synapsis and promote chiasma formation during C. elegans meiosis. Genes Dev 19: 2727-2743.

Moens PB, Tarsounas M, Morita $\mathrm{T}$, Habu T, Rottinghaus ST, Freire R, Jackson SP, Barlow C, Wynshaw-Boris A. 1999. The association of ATR protein with mouse meiotic chromosome cores. Chromosoma 108: 95-102.

Moens PB, Marcon E, Shore JS, Kochakpour N, Spyropoulos B. 2007. Initiation and resolution of interhomolog connections: Crossover and non-crossover sites along mouse synaptonemal complexes. J Cell Sci 120: 1017-1027.

Neale MJ, Pan J, Keeney S. 2005. Endonucleolytic processing of covalent protein-linked DNA double-strand breaks. Nature 436: 1053-1057.

Page SL, Hawley RS. 2003. Chromosome choreography: The meiotic ballet. Science 301: 785-789.

Perera D, Perez-Hidalgo L, Moens PB, Reini K, Lakin N, Syvaoja JE, San-Segundo PA, Freire R. 2004. TopBP1 and ATR colocalization at meiotic chromosomes: Role of TopBP1/Cut5 in the meiotic recombination checkpoint. Mol Biol Cell 15: 1568-1579.

Pittman DL, Cobb J, Schimenti KJ, Wilson LA, Cooper DM, Brignull E, Handel MA, Schimenti JC. 1998. Meiotic prophase arrest with failure of chromosome synapsis in mice deficient for Dmc1, a germline-specific RecA homolog. Mol Cell 1: 697-705.

Plug AW, Peters AH, Keegan KS, Hoekstra MF, de Boer P, Ashley T. 1998. Changes in protein composition of meiotic nodules during mammalian meiosis. J Cell Sci 111: 413-423.

Remenyi A, Good MC, Bhattacharyya RP, Lim WA. 2005. The role of docking interactions in mediating signaling input, output, and discrimination in the yeast MAPK network. Mol Cell 20: 951-962.

Reynard LN, Turner JM, Cocquet J, Mahadevaiah SK, Toure A, Hoog C, Burgoyne PS. 2007. Expression analysis of the mouse multi-copy X-linked gene Xlr-related, meiosis-regulated $(\mathrm{Xmr})$, reveals that Xmr encodes a spermatid-expressed cytoplasmic protein, SLX/XMR. Biol Reprod 77: 329-335.

Roig I, Dowdle JA, Toth A, de Rooij DG, Jasin M, Keeney S. 2010. Mouse TRIP13/PCH2 is required for recombination and normal higher-order chromosome structure during meiosis. PLoS Genet 6: e1001062. doi: 10.1371/journal.pgen. 1001062 . 
Romanienko PJ, Camerini-Otero RD. 2000. The mouse Spo11 gene is required for meiotic chromosome synapsis. Mol Cell 6: 975-987.

Royo H, Polikiewicz G, Mahadevaiah SK, Prosser H, Mitchell M, Bradley A, de Rooij DG, Burgoyne PS, Turner JM. 2010. Evidence that meiotic sex chromosome inactivation is essential for male fertility. Curr Biol 20: 2117-2123.

Shin YH, Choi Y, Erdin SU, Yatsenko SA, Kloc M, Yang F, Wang PJ, Meistrich ML, Rajkovic A. 2010. Hormadl mutation disrupts synaptonemal complex formation, recombination, and chromosome segregation in mammalian meiosis. PLOS Genet 6: e1001190. doi: 10.1371/journal.pgen.1001190.

Testa G, Schaft J, van der Hoeven F, Glaser S, Anastassiadis K, Zhang Y, Hermann T, Stremmel W, Stewart AF. 2004. A reliable lacZ expression reporter cassette for multipurpose, knockout-first alleles. Genesis 38: 151-158.

Turner JM, Aprelikova O, Xu X, Wang R, Kim S, Chandramouli GV, Barrett JC, Burgoyne PS, Deng CX. 2004. BRCA1, histone H2AX phosphorylation, and male meiotic sex chromosome inactivation. Curr Biol 14: 2135-2142.

Turner JM, Mahadevaiah SK, Fernandez-Capetillo O, Nussenzweig A, Xu X, Deng CX, Burgoyne PS. 2005. Silencing of unsynapsed meiotic chromosomes in the mouse. Nat Genet 37: 41-47.

Turner JM, Mahadevaiah SK, Ellis PJ, Mitchell MJ, Burgoyne PS. 2006. Pachytene asynapsis drives meiotic sex chromosome inactivation and leads to substantial postmeiotic repression in spermatids. Dev Cell 10: 521-529.

Wojtasz L, Daniel K, Roig I, Bolcun-Filas E, Xu H, Boonsanay V, Eckmann CR, Cooke HJ, Jasin M, Keeney S, et al. 2009. Mouse HORMAD1 and HORMAD2, two conserved meiotic chromosomal proteins, are depleted from synapsed chromosome axes with the help of TRIP13 AAA-ATPase. PLoS Genet 5: e1000702. doi: 10.1371/journal.pgen.1000702.

Yoshida K, Kondoh G, Matsuda Y, Habu T, Nishimune Y, Morita T. 1998. The mouse RecA-like gene Dmc1 is required for homologous chromosome synapsis during meiosis. Mol Cell 1: 707-718.

Zou L, Elledge SJ. 2003. Sensing DNA damage through ATRIP recognition of RPA-ssDNA complexes. Science 300: 1542-1548. 


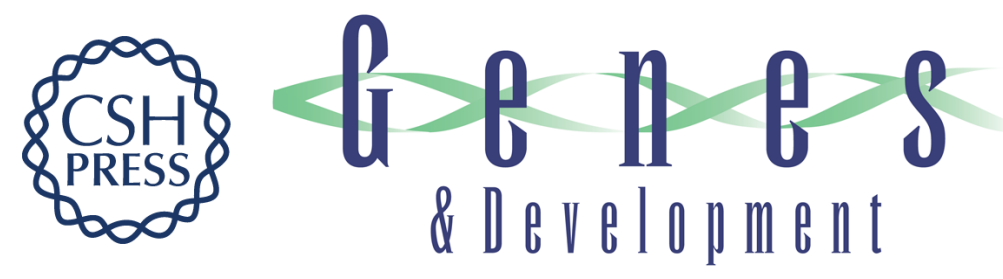

\section{Meiotic DNA double-strand breaks and chromosome asynapsis in mice are monitored by distinct HORMAD2-independent and -dependent mechanisms}

Lukasz Wojtasz, Jeffrey M. Cloutier, Marek Baumann, et al.

Genes Dev. 2012, 26:

Access the most recent version at doi:10.1101/gad.187559.112

Supplemental http://genesdev.cshlp.org/content/suppl/2012/04/25/26.9.958.DC1

Material

References This article cites 52 articles, 17 of which can be accessed free at: http://genesdev.cshlp.org/content/26/9/958.full.html\#ref-list-1

License

Email Alerting Receive free email alerts when new articles cite this article - sign up in the box at the top Service right corner of the article or click here.

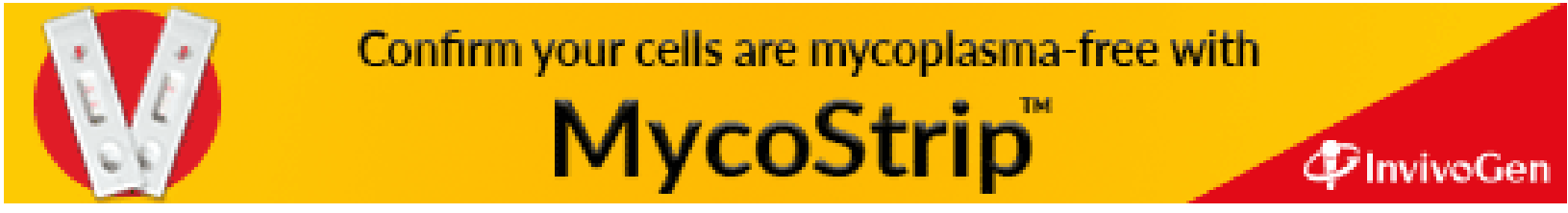

